\title{
Homotopy of exact coherent structures in plane shear flows
}

\author{
Fabian Waleffe ${ }^{\text {a) }}$ \\ Departments of Mathematics and Engineering Physics, University of Wisconsin-Madison, Madison, \\ Wisconsin 53706
}

(Received 13 August 2002; accepted 31 January 2003; published 2 May 2003)

\begin{abstract}
Three-dimensional steady states and traveling wave solutions of the Navier-Stokes equations are computed in plane Couette and Poiseuille flows with both free-slip and no-slip boundary conditions. They are calculated using Newton's method by continuation of solutions that bifurcate from a two-dimensional streaky flow then by smooth transformation (homotopy) from Couette to Poiseuille flow and from free-slip to no-slip boundary conditions. The structural and statistical connections between these solutions and turbulent flows are illustrated. Parametric studies are performed and the parameters leading to the lowest onset Reynolds numbers are determined. In all cases, the lowest onset Reynolds number corresponds to spanwise periods of about 100 wall units. In particular, the rigid-free plane Poiseuille flow traveling wave arises at $\operatorname{Re}_{\tau}=44.2$ for $L_{x}^{+}=273.7$ and $L_{z}^{+}$ $=105.5$, in excellent agreement with observations of the streak spacing. A simple one-dimensional map is proposed to illustrate the possible nature of the "hard" transition to shear turbulence and connections with the unstable exact coherent structures. (c) 2003 American Institute of Physics. [DOI: $10.1063 / 1.1566753]$
\end{abstract}

\section{INTRODUCTION}

A fundamental change in our understanding of the nature of shear turbulence started in the 1950s. Then the view of turbulence as the random interaction of "eddies" began to be replaced by one of organized motions interacting with the mean flow. Theodorsen ${ }^{1,2}$ proposed the qualitative, mechanistic picture of a wall-bound horsheshoe vortex as the fundamental structure in turbulent shear flows. His was a physically complete structure motivated by considerations of optimum streamwise enstrophy production. $\mathrm{Malkus}^{3}$ proposed a quantitative, nonmechanistic theory based on a principle of maximum energy dissipation rate, and marginal stability constraints, that led to the derivation of upper bounds and to Busse's picture of the optimum momentum transporting solenoidal field. ${ }^{4}$ That optimum field is more intricate but nonetheless similar to Townsend's qualitative "attached eddy" interpretation of two-point velocity correlation measurements. ${ }^{5,6}$ The upper bound fields and attached eddies are streamwise-invariant and better described as a combination of streamwise rolls and streaks. That combination will be called the "streaky flow" in Sec. IV. Townsend also introduced the qualitative concepts of "active" and "inactive" motions to describe turbulent shear flows. ${ }^{6}$

The experiments of Kline et al. ${ }^{7}$ first revealed the structure of near-wall turbulence and pointed to the importance of low-velocity streamwise streaks whose lift-up, oscillation and "bursting" were seen as the main turbulence producing mechanism. It was found that the streaks have a characteristic spacing of about 100 wall units (defined in Sec. VI). ${ }^{7,8}$ Those experiments sparked numerous experimental and computational studies, most of which are reviewed in the

${ }^{a)}$ Electronic mail: waleffe@ math.wisc.edu monograph edited by Panton. ${ }^{9}$ Another line of work based on the proper orthogonal decomposition is reviewed in the book by Holmes, Lumley, and Berkooz. ${ }^{10}$ Among all those contributions, the sketches of self-replicating horseshoe vortices in Acarlar and Smith ${ }^{11}$ and the, then unrelated, mean flow-first harmonic theory of Benney ${ }^{12}$ were the two key references that led to the present work. ${ }^{13,14}$ Benney's "mean flow" consists of weak streamwise rolls and a spanwise varying streamwise velocity, similar to Townsend's attached eddies.

It is now well accepted that the predominant vortex structure in the near-wall region consists not of horseshoe vortices but of staggered, quasi-streamwise vortices as in Stretch's sketch ${ }^{15}$ reproduced in Fig. 1. That picture is Stretch's synthesis of his pattern eduction studies of the Kim, Moin, and Moser data. ${ }^{16}$ A similar study and result can be found in Ref. 17. Horseshoe vortices and packets of such vortices are also observed in turbulent flows. ${ }^{18}$ Whether such structures are the varicose versions of the sinusoidal structures studied here or result from a dynamic self-organization of the staggered vortices is a matter for later study.

This paper reports on traveling wave solutions of the Navier-Stokes equations in plane Couette and Poiseuille flows with either given velocity (no-slip) or velocity derivative (slip) at the wall. Brief reports on this work have appeared in Refs. 19 and 20. The traveling waves travel at a constant velocity and are therefore steady in the proper Galilean frame. The propagation velocity is analogous to an eigenvalue and cannot be deduced a priori, except by symmetry in plane Couette flow. These solutions were obtained numerically using spectrally accurate finite approximations of the fields (Sec. II), based on Fourier expansion and Chebyshev integration (Sec. III), and Newton's method, not time integration. This is because the traveling waves are typically unstable from onset and therefore not directly ac- 


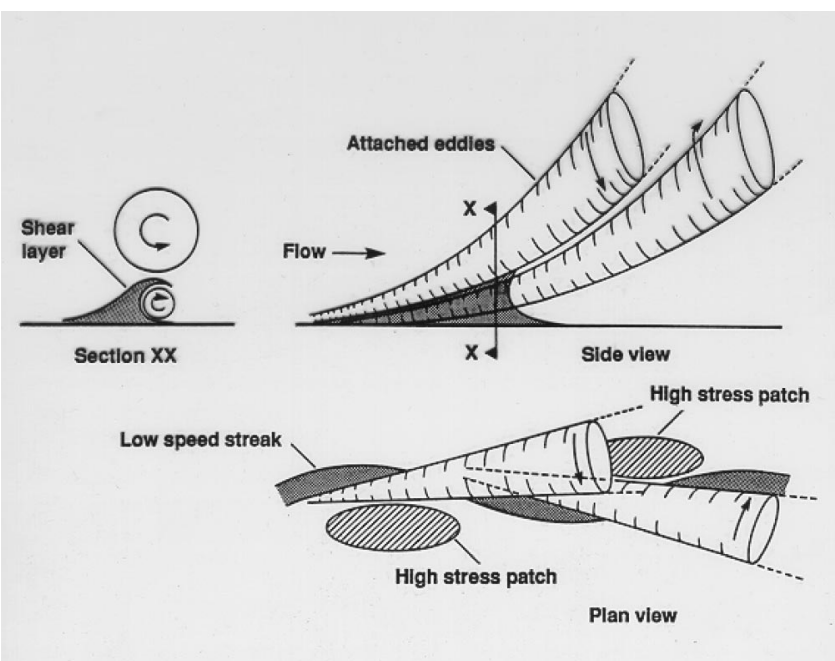

FIG. 1. Sketch of the coherent structure educed from DNS data (StretchRef. 15).

cessible to experiments and time-marching simulations. The traveling waves are remarkably similar to Stretch's picture of the typical coherent structure (Fig. 1). The latter is essentially an optimized ensemble average of significant regions of turbulent flow fields, localized in space and time, that filters out a spectrum of spatio-temporal fluctuations and does not satisfy the governing equations. In contrast, the traveling waves are solutions of the Navier-Stokes equations that propagate at constant speed without changing shape and are devoid of any other fluctuations. It is therefore appropriate to refer to the traveling wave solutions as "exact coherent structures." Furthermore, these exact coherent structures and their inherent instabilities are good candidates for precise definitions of "active" and "inactive" motions, respectively, that differ from Townsend's definitions. Townsend's active motions correspond to the momentum transporting streamwise rolls and streaks while his inactive motions would correspond to the streak instability. In fact, the streamwise ondulation, seen in Fig. 1 and traceable to the streak instability, is an essential element of the complete self-sustaining threedimensional (3-D) coherent structure.

The solutions were calculated using various continuation and homotopy procedures. The first step in this approach (Sec. IV) is based on a weakly nonlinear formulation of a fundamental self-sustaining process in shear flows. ${ }^{21-23}$ It consists in tracking the 3-D solutions that bifurcate from a self-consistent two-dimensional (2-D) flow made of streamwise rolls and streaks. The streamwise invariance of that base flow implies that it cannot be self-sustained, ${ }^{24}$ however, the streaks support an instability of inflectional type that feeds back on the rolls and leads to self-sustained, 3-D states. The full Navier-Stokes continuation in Sec. IV establishes a precise and explicit link between the 2-D streamwise invariant streaky flow ("attached eddies") and a 3-D self-sustained structure that consists of wavy streaks and staggered vortices, entirely similar to the observations.

Bifurcation from 2-D streaky flow can be used to compute 3-D self-sustained states in all plane shear flows but requires educated guessing of the streamwise rolls. Once the
3-D states have been found in one flow, it is simpler to extend them to other flows by homotopy. This consists in smoothly deforming ("morphing") the base flow into the desired flow while tracking the self-sustained solutions with Newton's method. Two types of homotopies are presented in Sec. V. One transforms free-slip into no-slip boundary conditions (12), the other transforms Couette flow into Poiseuille flow (27). These transformations are not only very effective at extending solutions to other boundary conditions and base states but also demonstrate the close connection (the homotopy or "same shape") between the various states. The homotopy between free-slip and no-slip demonstrates that noslip is not required for these exact coherent structures. The homotopies to no-slip plane Couette flow connects this broad family of exact coherent structures with the steady state solutions first computed in that flow by Nagata ${ }^{25}$ and studied by Clever and Busse. ${ }^{26,27}$

The exact coherent structures discussed here thus belong to a six-parameter $\left(\alpha, \gamma, \operatorname{Re}, \lambda_{b}, \lambda_{t}, \mu\right.$, defined below) family of three-dimensional vector fields that solve the Navier-Stokes equations. The solutions come in pairs, an upper branch and a lower branch, at a given Reynolds number. This is clearly a rich family and the 3-D illustrations in this paper are typically limited to the lowest-Re bifurcation point where the solutions first appear and where upper branch and lower branch coincide. A complete illustration of these solutions would require too many figures. The streamwise and spanwise length scales that lead to the lowest onset Reynolds for these exact coherent structures are presented in Sec. VI. The optimum spanwise length scales are all in the neighborhood of 100 wall units. In particular, the optimum spanwise length scale for the no-slip plane Poiseuille solution is $105.5^{+}$. This is the solution that is most relevant to the higher Reynolds number observations.

A few mean velocity and rms velocity fluctuation profiles of the exact coherent structures are presented in Sec. VII together with bifurcation diagrams for various parameters. Those results compare favorably with the statistics of turbulent flows suggesting that the exact coherent structures do indeed capture essential structural and statistical features of turbulent motions. But the exact coherent structures are steady in the appropriate Galilean frame and therefore have none of the disorder characteristic of turbulent flows. All these exact coherent structures also appear at Reynolds numbers that are significantly lower, typically a factor of 2 lower, than the smallest Reynolds numbers where turbulence is observed, and they are unstable from onset. How then, could these unstable exact coherent structures be of any relevance to the observations? The traveling waves in plane shear flows are not simply attractors for broad classes of initial conditions as in Fisher's equation, for instance, where the slowest wave is an attractor (see, e.g., Ref. 28). The traveling waves are most likely not solitons either as in the Korteweg-de Vries equation where broad classes of initial conditions evolve into a superposition of solitary traveling waves that preserve their shape through nonlinear interactions. The shear flow situation is more complex, but there is clear and increasing evidence that turbulent flows spend a lot of time "near" these exact coherent structures and therefore that 


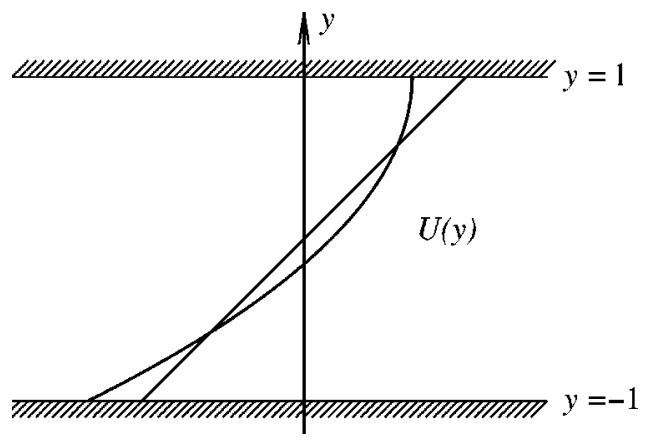

FIG. 2. Laminar base flows: Plane Couette $U(y)=y$ and plane Poiseuille $U(y)=y-y^{2} / 2+1 / 6$

much of the structure and statistics of turbulence can be understood from these traveling waves. Readers who are wellversed in nonlinear dynamics and chaos know the importance of unstable solutions. This paper ends with a onedimensional (1-D), discrete dynamical system that may be helpful as a simple concrete illustration of the nature of the "hard" transition to turbulence in shear flows and the relevance of unstable solutions.

\section{MATHEMATICAL FORMULATION}

The governing equations are the Navier-Stokes equations for incompressible flow,

$$
\frac{\partial \mathbf{v}}{\partial t}+\mathbf{v} \cdot \boldsymbol{\nabla} \mathbf{v}=-\boldsymbol{\nabla} p+\frac{1}{\operatorname{Re}} \nabla^{2} \mathbf{v}+\mathbf{F}, \quad \boldsymbol{\nabla} \cdot \mathbf{v}=0,
$$

where $\mathbf{v}$ is the Eulerian fluid velocity, $p$ the "pressure" (divided by the mass density), Re the Reynolds number. A Cartesian system of reference is employed with $x$ streamwise, $y$ shearwise (i.e., wall-normal) and $z$ spanwise. The corresponding velocity components are $u, v, w$, respectively. The flow is bounded by two infinite planes located at $y= \pm 1$ and is maintained by a body force $\mathbf{F}$ and/or the boundary conditions at $y= \pm 1$, where $\hat{\mathbf{x}}$ is the unit vector in the $x$ direction. The physical boundary conditions correspond to no-slip at the walls. These are Dirichlet boundary conditions where velocity is imposed on the boundary. In this paper, all three canonical types of boundary conditions, Dirichlet, Neumann, and Fourier-Robin [also called "of the third kind," see Eq. (12) below] are employed for the slip-to-no-slip homotopy. Periodicity is enforced in the streamwise and spanwise directions with periods $L_{x}=2 \pi / \alpha$ and $L_{z}=2 \pi / \gamma$, respectively.

Plane Couette and Poiseuille flows are considered. Plane Couette flow is maintained by the boundary conditions at $y$ $= \pm 1$ with no body force and has the laminar base solution $\mathbf{v}=y \hat{\mathbf{x}} \equiv \mathbf{U}_{L}^{(\mathrm{C})}$. Plane Poiseuille flow is maintained by an external uniform pressure gradient $\mathbf{F}=-\mathrm{Re}^{-1} \hat{\mathbf{x}}$, and the boundary conditions at $y= \pm 1$. The Poiseuille laminar base flow is chosen here as $\mathbf{v}=\left(y-y^{2} / 2+1 / 6\right) \hat{\mathbf{x}} \equiv \mathbf{U}_{L}^{(P)}$ (Fig. 2). This Poiseuille flow has zero average and its antisymmetric part is identical to the plane Couette base flow. This unusual Poiseuille normalization is chosen for the Couette-toPoiseuille homotopy. Therefore $y=+1$ is here a plane of reflection symmetry for plane Poiseuille flow with the boundary conditions $\partial u / \partial y=v=\partial w / \partial y=0$. The $y=1$ plane corresponds to the centerline of a full plane Poiseuille flow while $y=-1$ corresponds to the bottom wall.

The plane Couette flow Reynolds number implied by these normalizations is based on the half laminar wallvelocity difference and the half channel height. In free-slip (Neumann), this is equivalent to a Reynolds number based on the mean wall shear rate $d \bar{U} / d y= \pm 1$ at $y= \pm 1$. In noslip (Dirichlet), the laminar and total velocities at the wall are identical and the Reynolds number is therefore based on the half-wall velocity difference. For plane Poiseuille flow, the Reynolds number is based on half the distance between the wall and the centerline and half the difference between the laminar centerline velocity and the wall velocity. If $\ell$ is half the distance between the wall and the centerline (i.e., one-quarter of the channel height), $d P / d x$ the imposed (kinematic) pressure gradient, and $\nu$ is the kinematic viscosity then $\operatorname{Re}=|d P / d x| \ell^{3} / \nu^{2}$. Note that, in effect, the Reynolds numbers are based on the shear rate at the wall $S$ $\equiv|d \bar{U} / d y|_{\text {wall }}$, except for no-slip plane Couette flow. The friction velocity $u_{\tau} \equiv(\nu S)^{1 / 2}$ and the "wall unit" $\nu / u_{\tau}$ is thus well-defined a priori and directly related to the outer units through the Reynolds number. In no-slip plane Poiseuille flow, for instance, the nondimensional kinematic viscosity is $1 / \mathrm{Re}$, the nondimensional friction velocity is $u_{\tau}=(2 / \mathrm{Re})^{1 / 2}$, and wall-units are simply $(2 \mathrm{Re})^{1 / 2}$ times the nondimensional units. The friction Reynolds number in this case is $\operatorname{Re}_{\tau} \equiv 2 \ell u_{\tau} / \nu=(8 \mathrm{Re})^{1 / 2}$. Further details of the transformations to wall units are provided in Sec. VI.

The pressure gradient is eliminated by using the "rollstreak" projections of the momentum equations using the operators

$$
\mathbf{P}_{v} \equiv-\hat{\mathbf{y}} \cdot \boldsymbol{\nabla} \times(\boldsymbol{\nabla} \times(\cdot))
$$

and

$$
\mathbf{P}_{\eta} \equiv \hat{\mathbf{y}} \cdot \nabla \times(\cdot) .
$$

These projections lead to an equation for $\mathbf{P}_{v} \mathbf{v}=\nabla^{2} v$, the Laplacian of the $y$ velocity (where the continuity equation $\boldsymbol{\nabla} \cdot \mathbf{v}=0$ has been used) and another equation for $\mathbf{P}_{\eta} \mathbf{v}=\eta$, the $y$ vorticity. The $u$ and $w$ velocity components can be reconstructed using the definition of the $y$ vorticity, $\eta$ $=\partial u / \partial z-\partial w / \partial x$ and solenoidality $\boldsymbol{\nabla} \cdot \mathbf{v}=\partial u / \partial x+\partial v / \partial y$ $+\partial w / \partial z=0$. The two equations for $v$ and $\eta$ must be supplemented by equations for the $x$ and $z$ averaged velocities, $\bar{U}(y, t)$ and $\bar{W}(y, t)$. These are obtained by averaging the Navier-Stokes equations over $x$ and $z$.

This "roll-streak," or $v-\eta$, representation is closely linked to the "poloidal-toroidal" representation of the solenoidal velocity field:

$$
\mathbf{v}=\boldsymbol{\nabla} \times(\nabla \times \phi \hat{\mathbf{y}})+\nabla \times \psi \hat{\mathbf{y}}+\bar{U} \hat{\mathbf{x}}+\bar{W} \hat{\mathbf{z}}
$$

where $\phi=\phi(x, y, z, t)$ and $\psi=\psi(x, y, z, t)$ are threedimensional, time-dependent scalar fields while $\bar{U}=\bar{U}(y, t)$ and $\bar{W}=\bar{W}(y, t)$ are (1-D), time-dependent scalar fields (see, e.g., Refs. 25 and 26). Indeed, $v=-\left(\partial_{x}^{2}+\partial_{z}^{2}\right) \phi$ and $\eta$ 
$=-\left(\partial_{x}^{2}+\partial_{z}^{2}\right) \psi$. The roll (or poloidal) mode $(v, \phi)$ has no $y$-vorticity, while the streak (or toroidal) mode $(\eta, \psi)$ has no $y$ velocity.

In summary, the governing equations (1) are reduced to

$$
\begin{aligned}
& \left(\frac{\partial}{\partial t}-\frac{1}{\operatorname{Re}} \nabla^{2}\right) \nabla^{2} v+\mathbf{P}_{v} \cdot(\mathbf{v} \cdot \nabla \mathbf{v})=0, \\
& \left(\frac{\partial}{\partial t}-\frac{1}{\operatorname{Re}} \nabla^{2}\right) \eta+\mathbf{P}_{\eta} \cdot(\mathbf{v} \cdot \nabla \mathbf{v})=0, \\
& \left(\frac{\partial}{\partial t}-\frac{1}{\operatorname{Re}} \frac{\partial^{2}}{\partial y^{2}}\right) \bar{U}+\hat{\mathbf{x} \cdot(\mathbf{v} \cdot \boldsymbol{\nabla} \mathbf{v})}-\hat{\mathbf{x} \cdot \mathbf{F}}=0,
\end{aligned}
$$

where the overbar denotes an average over $x$ and $z$. The $\bar{W}(y, t)$ mean flow is identically zero by symmetry.

The full velocity field is decomposed into the laminar base flow plus a perturbation $\mathbf{v}=U_{L} \hat{\mathbf{x}}+\mathbf{u}$. In the following, $\mathbf{u}=(u, v, w)$ represents the perturbation from laminar flow unless otherwise noted. This paper is primarily concerned with traveling wave solutions of the form $\mathbf{u}(x, y, z, t)=\mathbf{u}(x$ $-C t, y, z, 0)$. Looking for such solutions is equivalent to looking for a three-dimensional vector field $\mathbf{u}(x, y, z)$ that satisfies the above-mentioned equations with $\partial_{t}$ replaced by $-C \partial_{x}$. This leads to the nonlinear eigenvalue problem

$$
\begin{aligned}
& \left(C \frac{\partial}{\partial x}+\frac{1}{\operatorname{Re}} \nabla^{2}\right) \nabla^{2} v-\mathbf{P}_{v} \cdot(\mathbf{v} \cdot \nabla \mathbf{v})=0, \\
& \left(C \frac{\partial}{\partial x}+\frac{1}{\operatorname{Re}} \nabla^{2}\right) \eta-\mathbf{P}_{\eta} \cdot(\mathbf{v} \cdot \nabla \mathbf{v})=0, \\
& \frac{1}{\operatorname{Re}} \frac{d^{2} \bar{u}}{d y^{2}}-\hat{\mathbf{x}} \cdot(\mathbf{v} \cdot \nabla \mathbf{v})=0,
\end{aligned}
$$

where the wave velocity $C$ is the eigenvalue. A unique solution is found by fixing the phase of the solution. Here we set

$$
\Im\langle\eta \exp (-i \alpha x)\rangle=0,
$$

where $\mathfrak{I}$ denotes imaginary part and $\langle\cdot\rangle$ a domain average.

The boundary conditions for the perturbation from laminar flow, u, are homogeneous. General slip boundary conditions of the form

$$
\lambda u-\kappa \partial_{n} u=v=\lambda w-\kappa \partial_{n} w=0,
$$

are employed at $y= \pm 1$, where $n$ is the direction normal to the wall into the fluid, $0 \leqslant \lambda \leqslant 1$ and $\kappa=1-\lambda$. In terms of $v$ and $\eta$ this translates into

$$
\begin{aligned}
& v=\lambda_{t} \partial_{y} v+\kappa_{t} \partial_{y}^{2} v=\lambda_{t} \eta+\kappa_{t} \partial_{y} \eta=0, \\
& v=\lambda_{b} \partial_{y} v-\kappa_{b} \partial_{y}^{2} v=\lambda_{b} \eta-\kappa_{b} \partial_{y} \eta=0
\end{aligned}
$$

at $y=+1$ and $y=-1$, respectively. Homotopy from freeslip to no-slip perturbations is performed by tracking solutions from $\lambda=0$ to $\lambda=1$. For Poiseuille flow, $\lambda_{t}=0$ always, as $y=1$ is the channel centerline. The Rayleigh-Bénard nomenclature "free-free," "rigid-free," and "rigid-rigid" is used to denote the boundary conditions at the bottom and top walls, respectively, with "free" indicating free-slip perturbations (Neumann boundary conditions) and "rigid" indicating no-slip perturbations (Dirichlet).

\section{NUMERICAL FORMULATION}

The primary variables $\bar{u}(y), v(x, y, z)$, and $w(x, y, z)$ are expanded in Fourier modes in the $x$ and $z$ directions and Chebyshev-based modes in the $y$ direction,

$$
\begin{aligned}
& v=\sum_{l=-L_{T}}^{L_{T}} \sum_{m=0}^{M_{T}} \sum_{n=-N_{T}}^{N_{T}} A_{l m n} e^{i l \alpha x} e^{i n \gamma z} \phi_{m}(y), \\
& \eta=\sum_{l=-L_{T}} \sum_{m=0}^{L_{T}} \sum_{n=-N_{T}}^{N_{T}} B_{l m n} e^{i l \alpha x} e^{i n \gamma z} \psi_{m}(y), \\
& \bar{u}=\sum_{m=0}^{M_{T}} \hat{u}_{m} \psi_{m}(y) .
\end{aligned}
$$

The $y$-expansion functions $\phi_{m}(y)$ and $\psi_{m}(y)$ are integrals of Chebyshev polynomials that satisfy the boundary conditions. The basic idea is to set $D^{4} \phi_{m}(y)=T_{m}(y)$ and $D^{2} \psi_{m}(y)=T_{m}(y) \quad$ where $\quad D \equiv d / d y \quad$ and $T_{m}(y)$ $=\cos (m \arccos y)$ is the Chebyshev polynomial of degree $m$. This approach leads to numerical operators that are well conditioned. ${ }^{29}$ After four $y$-integrations,

$$
\phi_{m}(y)=I^{4} T_{m}(y)+c_{0}+c_{1} y+c_{2} y^{2}+c_{3} y^{3},
$$

where $I=\int d y$ denotes indefinite $y$-integration. The constants $c_{i}$ are determined from the boundary conditions $\phi_{m}$ $=\lambda D \phi_{m} \pm \kappa D^{2} \phi_{m}=0$ at $y= \pm 1$ (13). This leads to welldefined $\phi_{m}(y)$ for $0 \leqslant \lambda \leqslant 1$.

To illustrate the validity and accuracy of this approach, consider the calculation of the least negative eigenvalue of the streamwise rolls problem,

$$
\left(D^{2}-\gamma^{2}\right)^{2} \hat{v}=\sigma\left(D^{2}-\gamma^{2}\right) \hat{v}
$$

with $\hat{v}( \pm 1)=D \hat{v}(-1)=D^{2} \hat{v}(1)=0$ (rigid-free). This is the linearized $v$-equation (5) for $x$-independent perturbations of the form $v(y, z, t)=e^{\sigma t} \hat{v}(y) \cos \gamma z$. It is a test case that is quite appropriate for this work. The $\phi_{m}$ expansion $\hat{v}$ $=\Sigma_{0}^{M_{T}} a_{m} \phi_{m}(y)$ is compared to a conventional Chebyshev expansion $\hat{v}=\Sigma_{0}^{M_{T}+4} b_{m} T_{m}(y)$, where the extra four polynomials in the latter case are used to impose the boundary conditions (Chebyshev-tau formulation). Equation (18) is projected onto the $M_{T}+1$ Chebyshev polynomials $T_{m}(y)$, $m=0, \ldots, M_{T}$, with the Chebyshev weight, by Gauss integration with at least $N_{p}=M_{T}+5$ Gauss points $y_{j}=\cos (2 j$ $-1) \pi /\left(2 N_{p}\right), j=1, \ldots, N_{p}$. Two Chebyshev-tau formulations are used. One formulation enforces the boundary conditions by eliminating $b_{M_{T}+1}, \ldots, b_{M_{T}+4}$ from the eigenvalue problem. The second formulation eliminates $b_{0}, \ldots, b_{3}$ and has smaller roundoff errors. The boundary conditions are automatically enforced by the $\phi_{m}$ expansion. For $\gamma=1.3$, the least negative eigenvalue is found to be $\sigma_{1}$ $=-5.990343885706669$ (using the $\phi_{m}$ expansion with $M_{T}=42$, somewhat arbitrarily). Figure 3 is a $\log -\log$ plot of the $\sigma_{1}$ error as a function of the resolution $M_{T}$. The $\phi_{m}$ formulation is stable and saturates at round-off level $\left(10^{-15}\right)$ while the Chebyshev-tau errors are plagued by roundoff errors of $O\left(M_{T}^{8}\right)$, as expected for (18). The eigenvalue of largest magnitude turns out to be positive for all three formula- 


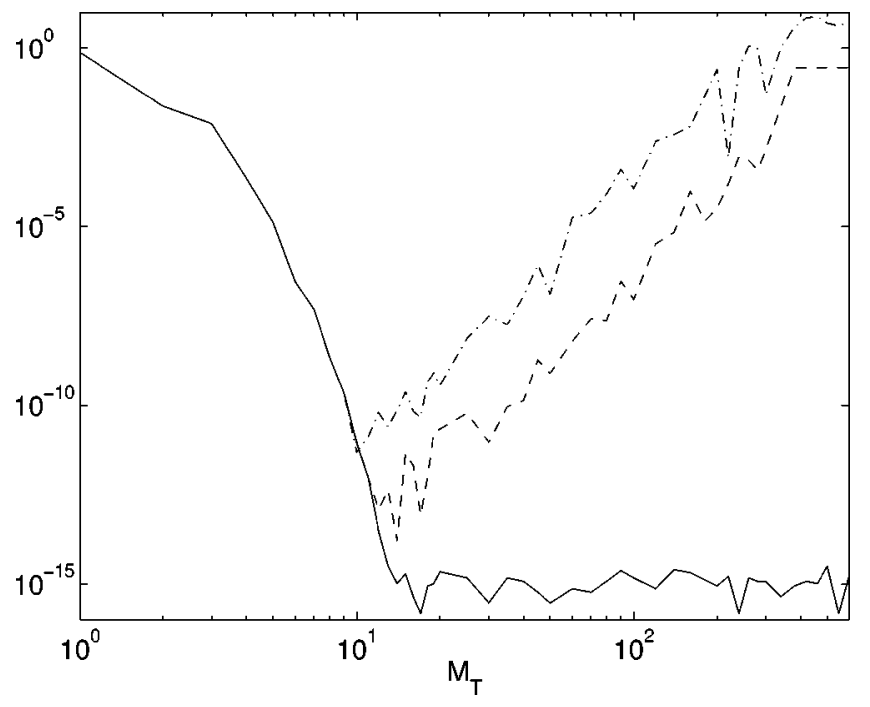

FIG. 3. Approximation error $\left|\tilde{\sigma}_{1}-\sigma_{1}\right| /\left|\sigma_{1}\right|$ where $\sigma_{1}$ is the least negative eigenvalue of (18). The $\phi_{m}$ expansion error (solid) saturates near $10^{-15}$. The Chebyshev-tau errors are $O\left(M_{T}^{8}\right)$ for $M_{T}>10$ (dash: $b_{0}, \ldots, b_{3}$ eliminated; dash-dot: $b_{M_{T}+1}, \ldots, b_{M_{T}+4}$ eliminated).

tions. That eigenvalue is spurious since it can be shown that the eigenvalues $\sigma$ in (18) are real and negative.

The definition of $\psi_{m}(y)$ is more delicate as the $D^{2}$ operator with slip boundary conditions $D \psi_{m}( \pm 1)=0$ is singular. To accommodate slip boundary conditions, we define $D^{2} \psi_{m}(y)=T_{m}(y)-\bar{T}_{m}$ for $m \neq 0$, where $\bar{T}_{m}$ $=\int_{-1}^{1} T_{m}(y) d y / 2$, yielding

$$
\psi_{m}(y)=I^{2} T_{m}(y)-\bar{T}_{m} y^{2} / 2+c_{0}+c_{1} y .
$$

The constants $c_{0}$ and $c_{1}$ are chosen to satisfy the boundary conditions $\lambda \psi_{m} \pm \kappa D \psi_{m}=0$ at $y= \pm 1$ (13). The constant $c_{0}$ is arbitrary in the free-free case $\left(\lambda_{t}=\lambda_{b}=0\right)$. In that case, we define $c_{0}=\lim _{\lambda_{b} \rightarrow 0} c_{0}\left(\lambda_{t}=0, \lambda_{b}\right)$ so the functions will be well adapted to the free-slip to no-slip homotopy. It turns out that $c_{0}$ is independent of $\lambda_{b}$ when $\lambda_{t}=0$. The zero mode is defined by $D^{2} \psi_{0}=\lambda_{t}+\lambda_{b}$. Two integrations and the boundary conditions yield

$$
\psi_{0}(y)=\left(\lambda_{t}+\lambda_{b}\right) \frac{y^{2}}{2}+\left(\lambda_{t}-\lambda_{b}\right) y+\frac{\lambda_{t}+\lambda_{b}}{2}-2 .
$$

For the full Navier-Stokes case, Eqs. (5)-(7), the nonlinear term is calculated by spectral convolution in the $x$ and $z$ directions but by collocation in the $y$ direction using at least $M_{T}+5$ Gauss points. The equations are projected onto the appropriate Chebyshev modes with the Chebyshev weight function. These projections are made by Gauss integration using at least $M_{T}+5$ Gauss points. All Fourier-Chebyshev modes with

$$
\frac{l^{2}}{\left(L_{T}+1\right)^{2}}+\frac{m^{2}}{\left(M_{T}+1\right)^{2}}+\frac{n^{2}}{\left(N_{T}+1\right)^{2}} \geqslant 1
$$

are truncated. This resolution approximately corresponds to a direct numerical simulation with resolution $\left(2 L_{T}+1\right)$
$\times\left(M_{T}+5\right) \times\left(2 N_{T}+1\right)$ after de-aliasing in $x$ and $z$. De-aliasing in $y$ was tested but made no difference on the scale of the plots shown here. There is no aliasing in $x$ and $z$.

The traveling wave nonlinear eigenvalue problem is solved using Newton iterations and various continuation and homotopy procedures, as discussed in later sections. The linear algebraic equations for $A_{l m n}, B_{l m n}$ and $\hat{u}_{m}(14)-(16)$ are solved with LAPACK routines. The code was thoroughly tested and all free-slip results obtained with a different triplyFourier code ${ }^{19}$ were reproduced with the present code. The linear instability of 2-D streaky flow ${ }^{22,23}$ was also reproduced as well as eigenmodes of the Orr-Sommerfeld and Squire operators. The size of the numerical problem is reduced by imposing the sinusoidal streak symmetry (24). Several resolutions have been used to verify numerical convergence. ${ }^{19,20}$ Most of the Poiseuille results presented here use $\left[L_{T}, M_{T}, N_{T}\right]=[11,23,11]$ corresponding to 7391 degrees of freedom. The optimum parameter results were checked with resolution $\left[L_{T}, M_{T}, N_{T}\right]=[13,25,13]$ for which there are 10977 degrees of freedom. Many of the Couette results enforce the additional symmetry (26) and $\left[L_{T}, M_{T}, N_{T}\right]$ up to $[15,27,15]$ (7697 modes) has been used.

\section{BIFURCATION FROM STREAKY FLOW}

\section{A. Self-sustaining process}

The physical mechanisms responsible for the coherent structures consist of a three-dimensional, nonlinear selfsustaining process. The process has been described and studied in several earlier references. ${ }^{21-23,30,31}$ The weakly nonlinear description of the process is that streamwise rolls $[0, V(y, z), W(y, z)]$ create a spanwise modulated shear flow $[U(y, z), 0,0]$ that is inflectionally unstable to a threedimensional perturbation $\exp (i \alpha x) \mathbf{u}(y, z)$. Here, $U(y, z)$ represents the total $x$-averaged streamwise velocity. The Reynolds stresses associated with that $3 \mathrm{D}$ perturbation extract energy from the spanwise fluctuation of the streamwise velocity $U(y, z)-\bar{U}(y)$, the streaks, but feed energy into the rolls and the mean shear $\bar{U}(y)$. This is along the lines of Benney's mean-flow first-harmonic theory, ${ }^{12}$ but here, viscous dissipation plays an important equilibrating role.

For $x$-independent flows, the streamwise rolls $[0, V(y, z), W(y, z)]$ decouple from the streamwise velocity, therefore they have no energy source and decay due to viscous dissipation. ${ }^{22,24}$ However, they redistribute the mean shear and can create substantial streaks. Indeed, rolls of $O\left(\mathrm{R}^{-1}\right)$ sustain $O(1)$ streaks. Quadratic nonlinear selfinteraction of an $O\left(\mathrm{Re}^{-1}\right) x$-dependent streak instability eigenmode is in turn sufficient to balance the viscous dissipation of the $O\left(\mathrm{Re}^{-1}\right)$ rolls. These scalings are for the lower branch of self-sustained states (see the following and Ref. 23, Sec. III D).

\section{B. Two-dimensional streaky flow}

Our method to construct exact coherent states in shear flows is to track solutions from a neutrally stable 2-D streaky 


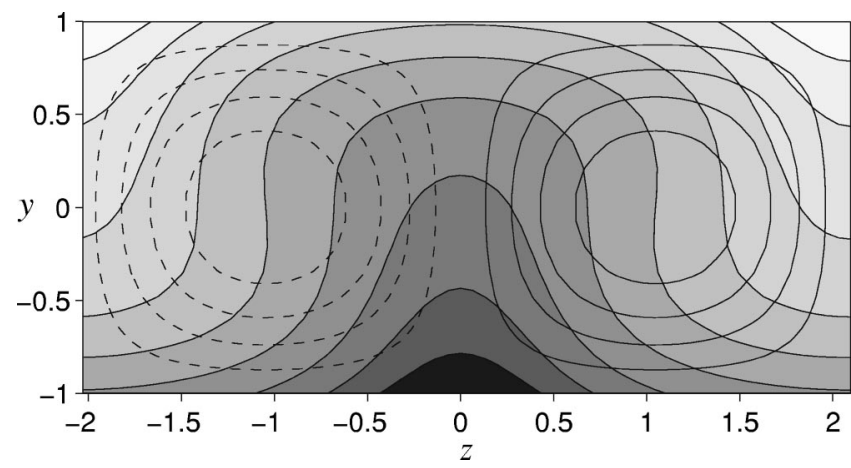

FIG. 4. Streaky flow $\mathbf{U}(y, z)$ for $F_{r}=5, \gamma=1.5$. Shaded contours of $U(y, z)$ at multiples of $0.1(\max U-\min U)=0.1703$ with contours of $\omega_{x}$ at multiples of $0.2 \max \omega_{x}=0.0208$. Positive $\omega_{x}$ contours solid, negative dashed.

flow $[U(y, z), V(y, z), W(y, z)]$, with externally maintained rolls of $O\left(\mathrm{Re}^{-1}\right)$, to a self-sustained fully 3-D flow. ${ }^{19}$ This is most easily done in plane Couette flow with free-slip boundary conditions where appropriate streamwise rolls have the simple form $V(y, z)=\operatorname{Re}^{-1} F_{r} \cos \beta y \cos \gamma z$, with $\beta=\pi / 2$ and $W(y, z)$ from continuity. Such rolls are an exact nonlinear solution and can be maintained by the weak $O\left(\mathrm{Re}^{-2}\right)$ external body force,

$$
\mathbf{F}=\frac{F_{r}}{\operatorname{Re}^{2}} \frac{\beta^{2}+\gamma^{2}}{\gamma}\left(\begin{array}{c}
0 \\
\gamma \cos \beta y \cos \gamma z \\
\beta \sin \beta y \sin \gamma z
\end{array}\right) .
$$

The streamwise velocity $U(y, z)$ results from a balance between viscous damping and redistribution by the rolls of the shear supplied at the walls as governed by the advectiondiffusion equation

$$
V \frac{\partial U}{\partial y}+W \frac{\partial U}{\partial z}=\frac{1}{\operatorname{Re}} \nabla^{2} U .
$$

Note that $V=O\left(\mathrm{Re}^{-1}\right)$ implies that $U(y, z)$ is Reynolds number independent and the streaks are indeed $O(1)$. A more precise estimate of the necessary rolls is obtained by balancing the advection of the laminar shear $d U_{L} / d y=1$ with the viscous damping of $O(1)$ streaks $V \approx\left(\beta^{2}\right.$ $\left.+\gamma^{2}\right) / \operatorname{Re}$, yielding $F_{r} \approx \beta^{2}+\gamma^{2}$. We expect that the optimum rolls have approximately unit aspect ratio and choose $\gamma=1.5$. Smaller $\gamma$ would produce weaker spanwise shear and therefore less vigorous sustenance of the 3-D streak eigenmode, while larger $\gamma$ leads to stronger dissipation of the rolls and the streaks. For $\gamma=1.5$ and $\beta=\pi / 2, F_{r} \approx 4.7$. For the Reynolds number, it must be low enough that rolls occupy the full channel but not so low that viscosity wipes out all perturbations from laminar. A trial value of $\mathrm{Re}=150$ was selected. The resulting two-dimensional three-component (2D-3C) flow $\mathbf{U}(y, z)=[U(y, z), V(y, z), W(y, z)]$ is depicted in Fig. 4, which shows contours of $U(y, z)$ overlayed with contours of streamwise vorticity $\omega_{x}=\partial W / \partial y-\partial V / \partial z$ for $F_{r}=5$ and $\operatorname{Re}=150$. The weak rolls indeed lead to a major redistribution of streamwise velocity. The mean velocity profile (not shown) has an S shape typical of turbulent Couette flow with a total mean shear at $y=0$ of $d \bar{U} / d y=0.093$ and a wall velocity at $y=1$ of $\bar{U}=0.434$, both down from their laminar value of 1 .

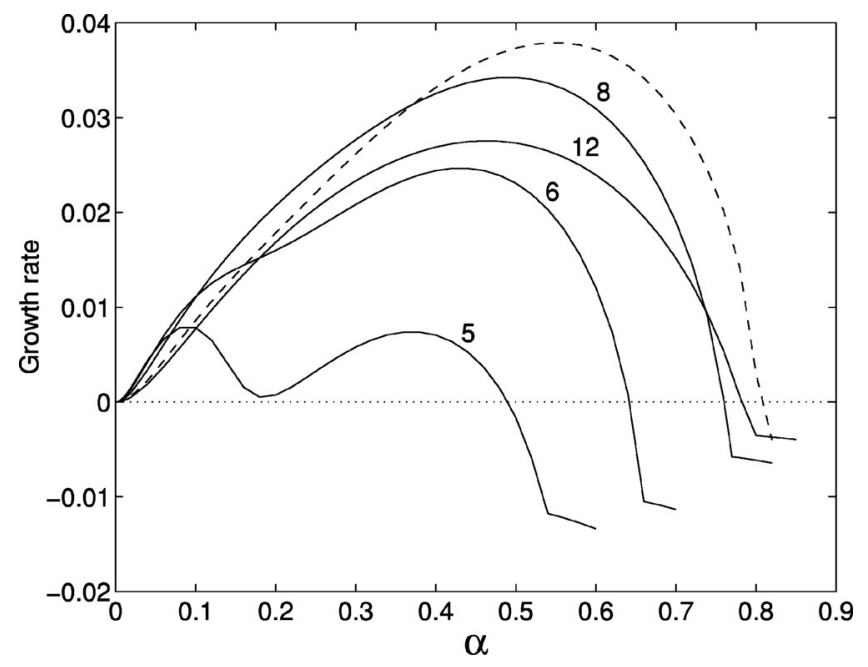

FIG. 5. Instability of streaky flow $[U(y, z), V(y, z), W(y, z)]$ with $\gamma=1.5$ for $F_{r}=5,6,8$, and 12. Dashed line is instability of $[U(y, z), 0,0]$ for $F_{r}$ $=8$.

\section{Instability of streaky flow}

The streaky flow $[U(y, z), V(y, z), W(y, z)]$ is unstable to $x$-dependent perturbations as a result of the strong spanwise inflections in $U(y, z)$. Three modes of instability with distinct symmetries (fundamental sinusoidal, fundamental varicose and subharmonic "sinucose") are possible. ${ }^{22,23}$ Previous work ${ }^{22,23}$ indicates that the fundamental sinusoidal mode is the most unstable (see also Ref. 32). That mode corresponds to the shift-reflection symmetry

$$
\left(\begin{array}{c}
u \\
v \\
w
\end{array}\right)(x, y, z)=\left(\begin{array}{c}
u \\
v \\
-w
\end{array}\right)\left(x+\frac{L_{x}}{2}, y,-z\right) .
$$

The Navier-Stokes equations linearized about the streaky flow $\mathbf{U}(y, z)$ admit separable solutions of the form $e^{\sigma t} e^{i \alpha x} \mathbf{u}(y, z)$. Figure 5 shows the growth rate, max $\mathfrak{R}(\sigma)$, as a function of the streamwise wave number $\alpha$ for streaky flows corresponding to $F_{r}=5,6,8,12$ at $\mathrm{Re}=150$. At this Reynolds number, the most unstable streaky flow corresponds to $F_{r} \approx 8$. For larger values of $F_{r}$, the rolls transport momentum faster than it can be supplied at the walls, the streamwise velocity $U(y, z)$ therefore weakens and with it the streak instability. For lower values of $F_{r}$ the rolls are not strong enough compared to streak dissipation, the streaks are weak and the mean shear is strong, resulting also in a collapse of the streak instability. The minimum $F_{r}$ sustaining a streaky flow instability is approximately $F_{r}=5$ at $\mathrm{Re}=150$. The growth rate of the instability for the pure streaky flow (no rolls) when $F_{r}=8$ is shown as a dashed line in Fig. 5. That curve indicates that the rolls reduce the growth rate for that streaky flow when $\alpha>0.375$, suggesting that the streak instability feeds energy back into the rolls for those $\alpha$ 's. Removing the mean shear leads to a tripling of the maximum growth rate and a doubling of the band of unstable wave numbers (Fig. 4 in Ref. 31) indicating that the mean shear has a strong stabilizing effect and that the instability originates in the spanwise inflections of $U(y, z)$. For more mar- 


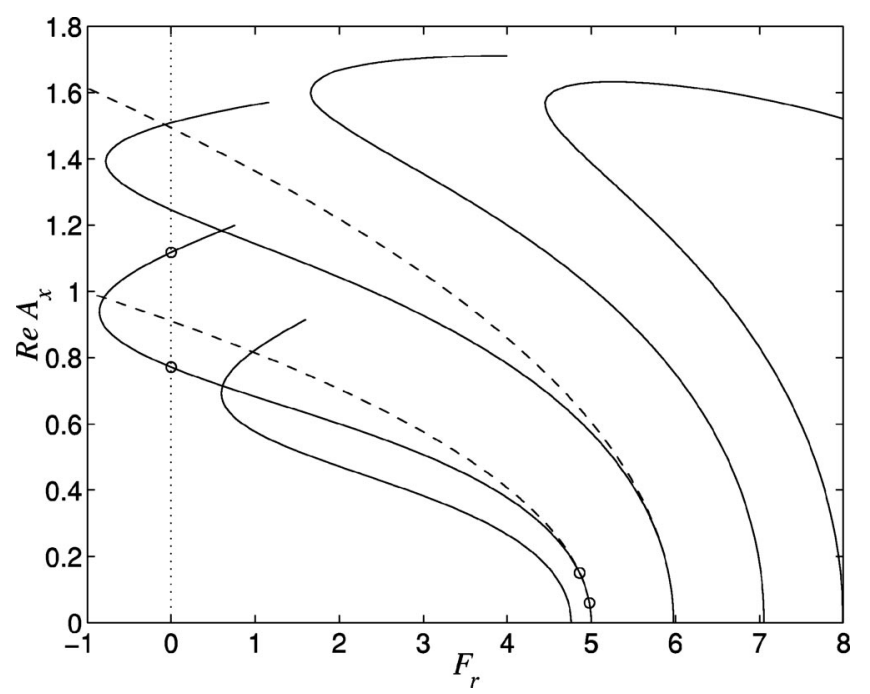

FIG. 6. Bifurcations from streaky flow in free-free plane Couette flow for $\gamma=1.5, \mathrm{Re}=150$, and $\alpha=0.4,0.49,0.64,0.72,0.76$ originate at $A_{x}=0$ near $F_{r}=4.76,5,6,7$, and 8, respectively. Dashed lines: $F_{r}=5-6.03\left(\operatorname{Re} A_{x}\right)^{2}$ and $F_{r}=5.98-2.68\left(\operatorname{Re} A_{x}\right)^{2}$. Open circles on $\alpha=0.49$ curve correspond to Fig. 7.

ginal values of $F_{r}$, e.g., $F_{r}=5$, the rolls have a strong effect on the structure of the eigenmodes and removing them changes the character of the instability.

\section{Continuation from streaky flow}

Our goal is to calculate nontrivial 3-D self-sustained states at $F_{r}=0$ so we select a weak forcing that still sustains unstable streaks. The streaky flow corresponding to $F_{r}=5$ is neutrally stable at $\alpha \approx 0.49$ (Fig. 5), hence a 3-D steady solution bifurcates from the 2-D streaky flow at $F_{r} \approx 5$, Re $=150, \alpha=0.49$. That branch of 3-D solutions is tracked by letting $F_{r}$ evolve as a state variable with a suitable measure of the 3-D state, $A_{x}$, as the control parameter. Here

$$
A_{x}=\Re\langle\eta \exp (-i \alpha x)\rangle
$$

is the $y$ average of the real part of the $l=1, n=0, \eta$-mode (15). This choice is made because of the predominant role of that mode in the streak instability. ${ }^{22}$ This $A_{x}$ definition provides the supplementary equation needed to determine the free variable $F_{r}$. The $A_{x}$ used here is $\alpha / \sqrt{2}$ times that used in Ref. 19. In summary, we look for a solution of the nonlinear system of equations (8)-(11) with the extra roll forcing term $\operatorname{Re}^{-2} F_{r}\left(\beta^{2}+\gamma^{2}\right)^{2} \cos \beta y \cos \gamma z$ on the right-hand side of (8) together with the additional equation (25). That solution is calculated by continuation in the control parameter $A_{x}$ starting from the neutrally stable streaky flow solution at $A_{x}=0$.

The $F_{r}-A_{x}$ bifurcation curve is shown in Fig. 6 and the bifurcating solution is illustrated in Fig. 7. The dashed lines in Fig. 6 are the fits $F_{r}=c_{0}+c_{2}\left(\operatorname{Re} A_{x}\right)^{2}$ near the bifurcation points at $A_{x}=0$. The Re factor is included because an $A_{x}$ of $O\left(\mathrm{Re}^{-1}\right)$ is expected for self-sustenance (Sec. IV A). The coefficient $c_{2}$ is strictly negative, verifying that the quadratic nonlinear self-interaction of the neutral streaky flow eigenmode has positive feedback on the rolls. These results can be obtained from a weakly nonlinear analysis of streaky flow as sketched in Refs. 22 and 23. The $F_{r}$ needed to maintain the rolls decreases as $A_{x}$ increases leading to two self-sustained solutions, a lower branch at $\operatorname{Re} A_{x}=0.7716$ [Fig. 7(c)] and an upper branch at $\operatorname{Re} A_{x}=1.1181$ [Fig. 7(d)]. The solid line in Fig. 6 is the fully nonlinear steady state, very well-resolved at $L_{T}=9, M_{T}=21, N_{T}=9$. Figure 6 also shows the solutions for $\alpha=0.40,0.64,0.72$, and 0.76 that bifurcate from $A_{x}$ $=0$ near $F_{r}=4.76,6,7$, and 8, respectively. There does not seem to be any bifurcation below the value $F_{r} \approx 4.7$ estimated in Sec. IV B. First-order nonlinear feedback on the rolls therefore is a general characteristic of unstable streaky flows. However, comparing Figs. 5 and 6 shows that bifurcation from the most unstable streaky flow does not lead to self-sustained steady states. The curves eventually turn back down and reintercept the $A_{x}=0$ axis near $F_{r}=13$ for $\alpha$ $=0.76, F_{r}=16$ for $\alpha=0.64$ and 18.4 for $\alpha=0.49$, for example.

Figure 7 shows the isosurfaces of $\omega_{x}$ $= \pm 0.80 \mathrm{max} \omega_{x}(x, y, z)$, corresponding to the innermost vorticity contours in Fig. 4, overlayed with the streamwise velocity isosurface corresponding to $u=\min u(x, y=0, z)$. Four $A_{x}$ values are shown, $\operatorname{Re} A_{x}=0.06,0.15,0.7716$, and 1.1181. The latter two correspond, respectively, to the lower branch and upper branch of self-sustained solutions with $F_{r}$ $=0$. As $A_{x}$ is increased, the weak streamwise rolls are first affected. The $\omega_{x}$ isosurfaces pinch off to form staggered vortices whose $x$-oriented axes are pointing up and away from the low-speed streak (in this plane Couette flow there is an equally strong high-speed streak centered at $z=\pi / \gamma$ that is not shown). As $A_{x}$ is increased further, the streak develops a strong undulation in the $x$ direction and the top tips of the vorticity isosurfaces move back toward the streak. The structure of these self-sustained free-free plane Couette flow solutions is very similar that of the coherent structures observed in the near-wall region of no-slip turbulent flows. ${ }^{15,17}$ The plane Couette 3-D solutions travel at the average flow velocity and have the additional shift-rotation symmetry ${ }^{22,23}$

$$
\left(\begin{array}{c}
u \\
v \\
w
\end{array}\right)(x, y, z)=\left(\begin{array}{c}
-u \\
-v \\
w
\end{array}\right)\left(\frac{L_{x}}{2}-x,-y, \frac{L_{z}}{2}+z\right) .
$$

This symmetry results from the $\pi$ rotation about the spanwise axis symmetry of plane Couette flow together with the $x$-phase choice imposed by $\Im\langle\eta \exp -i \alpha x\rangle=0$ and the $z$-phase choice imposed by (24). The symmetries (24) and (26) imply a reflection symmetry about the point $\left(0,0, L_{z} / 4\right): \mathbf{u}(x, y, z)$ $=-\mathbf{u}\left(-x,-y, L_{z} / 2-z\right)$. The symmetry (26) was imposed in a triply Fourier code ${ }^{19}$ and is also imposed for most of the Couette results show here.

\section{E. Continuation of self-sustained states}

The self-sustained solutions can be continued to different values of the parameters $\alpha, \gamma$, and Re. The Re continuations have been performed typically with a logarithmic arclength continuation procedure in $\operatorname{Re}-A_{x}$ space that uses the supplementary equations $\operatorname{Re}=R_{0}(1+\epsilon \cos \theta)$ and $A_{x}$ $\equiv \Re\langle\eta \exp (-i \alpha x)\rangle=A_{0}(1+\epsilon \sin \theta)$ to determine Re and $\theta$ for 

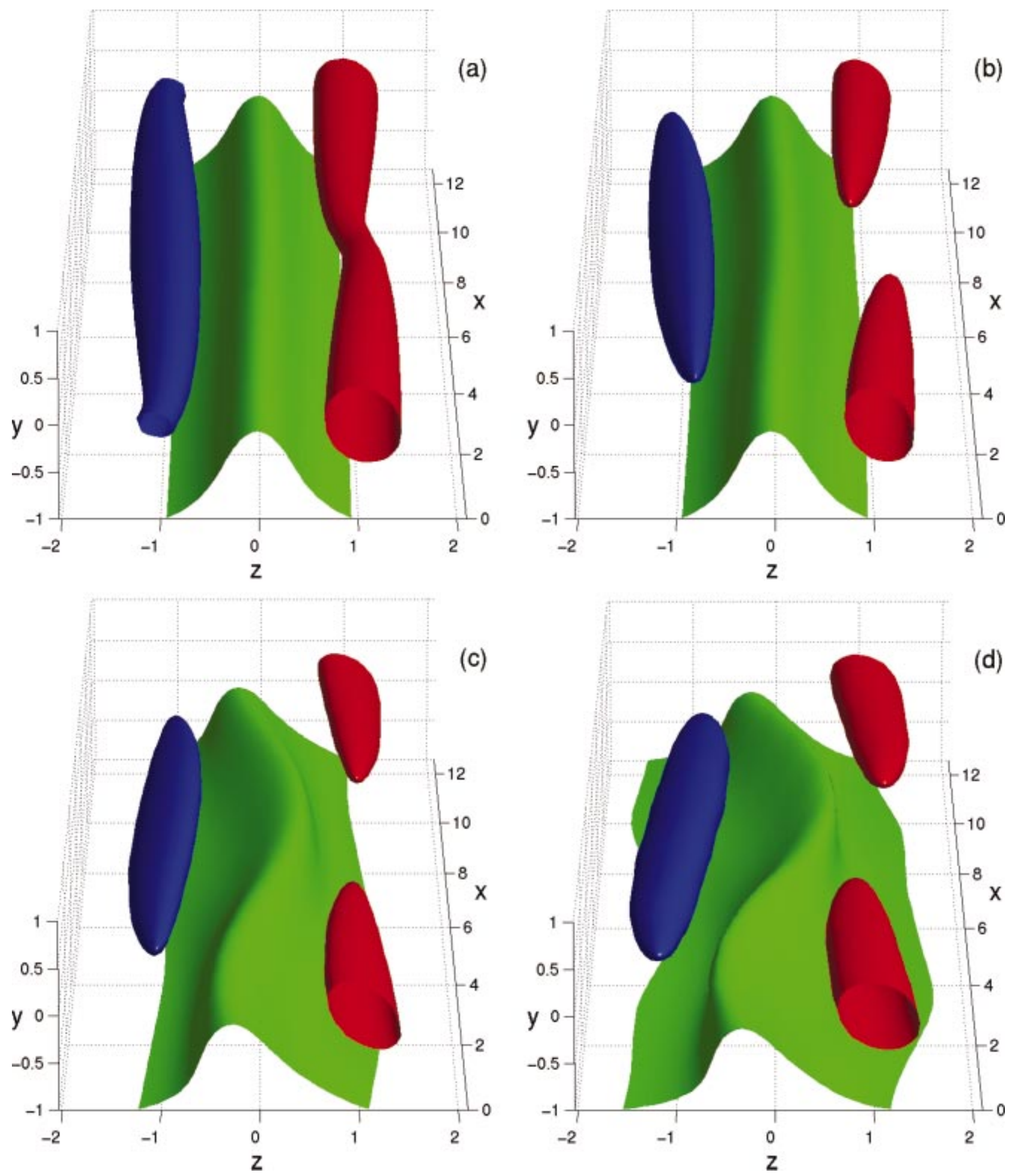

FIG. 7. (Color) Bifurcation from 2-D streaky flow at $\operatorname{Re}=150, \alpha=0.49, \gamma=1.5$, (a) $\operatorname{Re} A_{x}=0.06$, (b) $\operatorname{Re} A_{x}=0.15, \quad$ (c) $\operatorname{Re} A_{x}=0.7716, \quad$ (d) $\operatorname{Re} A_{x}$ $=1.1181$. Isosurface of $u=\min u(x, y=0, z)$ (green), $\omega_{x}=-0.8 \max \omega_{x}$ (blue), $\omega_{x}=0.8 \max \omega_{x}$ (red).

given $A_{0}, R_{0}$, and $\epsilon \ll 1$. The $\alpha$ and $\gamma$ continuations have been performed at fixed Re or $A_{x}$. When $F_{r}=0$, as will be the case for all results hereafter, the three-dimensional solutions arise through a saddle-node bifurcation at $\operatorname{Re}=\operatorname{Re}_{\mathrm{sn}}(\alpha, \gamma)$, the nose of the curves in Fig. 8. The solutions cease to exist for $\mathrm{Re}$ below $\mathrm{Re}_{\mathrm{sn}}$. A search for the lowest Reynolds number where these solutions first appear has been made by minimizing Re over $\alpha, \gamma$, and $A_{x}$. The optimum parameters for free-free Couette are $\alpha=0.3236$, $\gamma=0.7389$, for which the self-sustained solutions first appear at $\mathrm{Re}=99.9795$. The bump in the $\mathrm{Re}-A_{x}$ curve shown in Fig. 8 for $\alpha=0.5, \gamma=1.5$, corresponds to a splitting of the vortices. For Reynolds numbers near $\operatorname{Re}_{s n}=141.5951$, the $\omega_{x}$ vorticity maximum of 0.3661 occurs at $x=0, y=0, \gamma z$ 

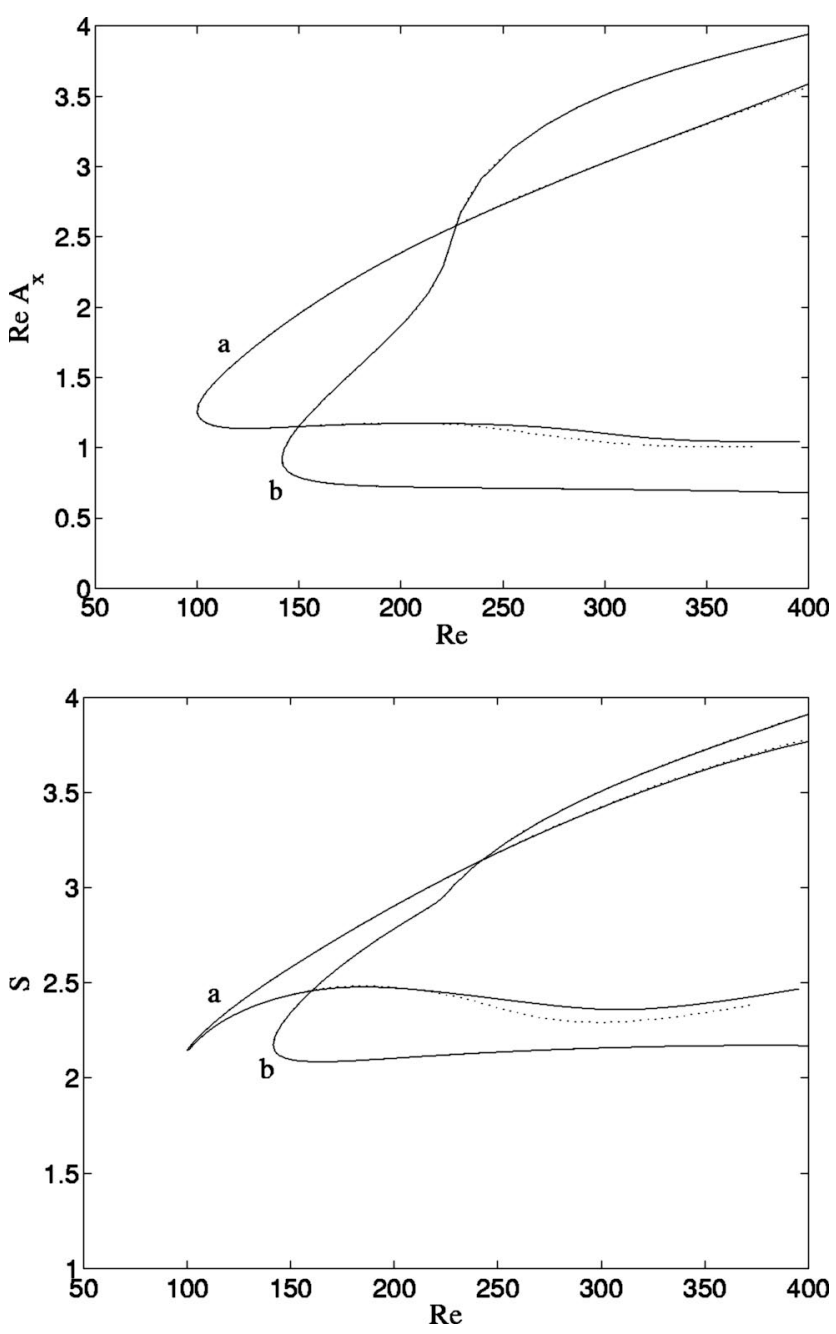

FIG. 8. Bifurcation diagrams for free-free plane Couette flow. Top: $A_{x}[\mathrm{Eq}$. (25)] vs Reynolds number Re. Bottom: Wall shear rate $S$ normalized by its laminar value for the same wall-velocity difference $[S=2 /(\bar{U}(1)$ $-\bar{U}(-1))$ in our units] vs Re. Curves labeled (a) $\alpha=0.3236, \gamma=0.7389$ yielding the lowest $\mathrm{Re}_{\mathrm{sn}}=99.9795$ (solid: $\left[L_{T}, M_{T}, N_{T}\right]=[15,27,15]$, dot: $[13,25,13]) . \quad$ Label (b): $\quad \alpha=0.5, \quad \gamma=1.5 \quad$ with $\quad \operatorname{Re}_{\mathrm{sn}}=141.5951$, $\left(\left[L_{T}, M_{T}, N_{T}\right]=[13,25,13]\right.$ and $[11,23,11]$ overlap $)$.

$=\pi / 2$ for $\alpha=0.5, \gamma=1.5$. The maximum stays at that location as $\operatorname{Re}$ is increased at fixed $\alpha, \gamma$, up until the bump in the $\operatorname{Re}-A_{x}$ curve where it splits into two equal maxima whose locations are symmetric about the point $x=0, y=0, \gamma z$ $=\pi / 2$ and Reynolds number dependent. This indicates the splitting of the vortices into two pairs, one pair associated with the upper wall streaks and one with the lower wall. For the optimum parameters $\alpha=0.3236$ and $\gamma=0.7389$, the vorticity maximum is split from the onset of the threedimensional solutions at $\mathrm{Re}_{\mathrm{sn}}=99.9795$. Bumps in the $\operatorname{Re}-A_{x}$ curves typically correspond to such splitting of the vortices or a harmonic modulation of the streaks.

\section{HOMOTOPY OF COHERENT STATES}

The continuation from 2-D streaky flow can be used to compute self-sustained 3-D solutions in no-slip plane Couette and plane Poiseuille flow. This was performed successfully for free-free plane Poiseuille flow (Waleffe 1998, un- published) but required some fiddling to determine the appropriate streamwise rolls. Two Fourier modes in $y$ were needed instead of the single mode in free-free Couette (22). The reason for this is that the appropriate streamwise rolls in plane Poiseuille flow turn out to be more concentrated away from the wall than in plane Couette flow. Continuation from 2-D streaky flow in no-slip plane Couette was initiated in Refs. 22 and 23.

A more robust and elegant approach is to let Navier, Stokes, and Newton select the appropriate streamwise rolls. This is done by homotopy from plane Couette to plane Poiseuille flow and from free-slip to no-slip. The Couette to Poiseuille homotopy consists of tracking the 3-D traveling wave solutions for the laminar flow

$$
U_{L}(y)=y+\mu\left(\frac{1}{6}-\frac{y^{2}}{2}\right)
$$

from $\mu=0$ to $\mu=1$. The free-slip to no-slip homotopy consists of tracking the traveling waves from $\lambda=0$ to $\lambda=1$ in the general slip boundary conditions (13). There is a $\lambda$ for each boundary, $\lambda_{t}$ for $y=+1$ and $\lambda_{b}$ for $y=-1$. All three homotopies are easily achieved. Steps of 0.1 in $\mu$ and/or $\lambda$ have been chosen arbitrarily. The free-free Couette solutions have been mapped into free-free Poiseuille traveling waves. The rigid-free traveling waves have been computed successfully by homotopy from both free-free Poiseuille as well as from free-free Couette. The rigid-rigid Couette solution was obtained by homotopy from a rigid-free Poiseuille solution only, because of a technical choice in the definition of the $\psi_{m}(y)$ functions (19). These homotopies are devices to obtain the various solutions using Newton's method but they also provide explicit demonstrations that all these threedimensional Navier-Stokes solutions can be smoothly deformed into one another.

The traveling wave solutions for the four basic flows, free-free and rigid-rigid plane Couette and free-free and rigid-free plane Poiseuille, are compared and illustrated in Figs. 9-11, for the parameters $\alpha=0.5, \gamma=1.5$ at their respective critical Reynolds number $\operatorname{Re}_{\mathrm{sn}}$ where the upper branch and lower branch solutions coalesce. The comparison is restricted to those parameters to limit the number of figures but it must be emphasized that the structure of the solutions varies with the parameters. The various solutions are clearly very similar to one another. They all correspond to a wavy low-speed streak flanked by staggered quasistreamwise vortices. The Couette solutions are fixed points in this frame of reference and have the additional symmetry (26). The free-free Couette $\operatorname{Re}_{\mathrm{sn}}=141.6$ and the rigid-rigid Couette $\operatorname{Re}_{\mathrm{sn}}=163.4$. The Poiseuille solutions are traveling waves with $C-\bar{U}(-1)=0.7413, \bar{U}(1)-\bar{U}(-1)=0.8802$, for the free-free solution at $\operatorname{Re}_{\mathrm{sn}}=156.4$ and $C-\bar{U}(-1)$ $=1.0380, \bar{U}(1)-\bar{U}(-1)=1.1392$, for the rigid-free solution at $\operatorname{Re}_{\mathrm{sn}}=251.5$. The main difference between free-slip and no-slip boundary conditions can be characterized as an "extra" small layer near the wall in the no-slip case. That "viscous sublayer" is about 5 wall units. The similarities between no-slip and free-slip solutions are further illustrated in a crude way in Figs. 12 and 13 where the mean velocity 

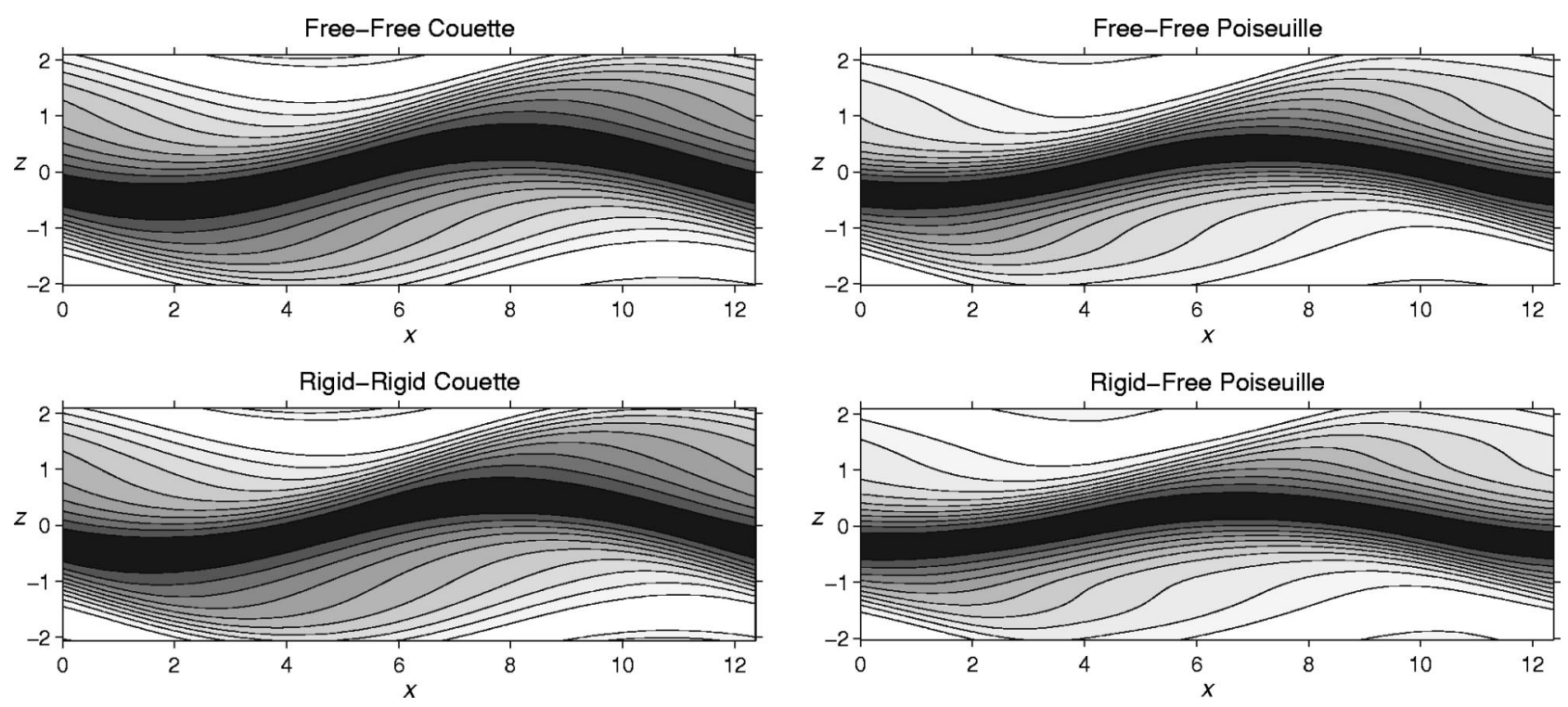

FIG. 9. Contours of streamwise velocity $u$ at $y=0$ for ECS at $\alpha=0.5, \gamma=1.5$, and $\operatorname{Re}_{\mathrm{sn}}=141.6$ (free-free Couette), $\operatorname{Re}_{\mathrm{sn}}=156.4$ (free-free Poiseuille), $\mathrm{Re}_{\mathrm{sn}}=163.4$ (rigid-rigid Couette), $\mathrm{Re}_{\mathrm{sn}}=251.5$ (rigid-free Poiseuille).

profiles are shown together with a no-slip profile that has been stretched to remove the viscous sublayer. The plane Couette no-slip mean profile is shown stretched uniformly in $y$ about the channel centerline at $y=0$ (Fig. 12). The stretching $y \rightarrow 1.5237 y$ is selected to match the free-slip velocities at the walls $\bar{U}( \pm 1)=0.4598$. The plane Poiseuille no-slip mean profile is shown stretched uniformly about the full channel centerline at $y=1$. The stretching $y \rightarrow 1+1.0721(y$ $-1)$ is selected to match the free-slip velocity difference
$\bar{U}(1)-\bar{U}(-1)=0.8802$. A more refined comparison between the two types of boundary conditions would seek the no-slip solutions for horizontal dimensions such that the aspect ratios of the no-slip inner layers match that of the full free-slip solutions (e.g., $\alpha \approx 0.75, \quad \gamma \approx 2.25$ for rigid-rigid Couette). As noted in Refs. 19 and 20, the Poiseuille mean profiles have two inflection points. The wave velocity $C$ is almost identical to the mean velocity at the inflection point that corresponds to a local minimum of mean shear rate.
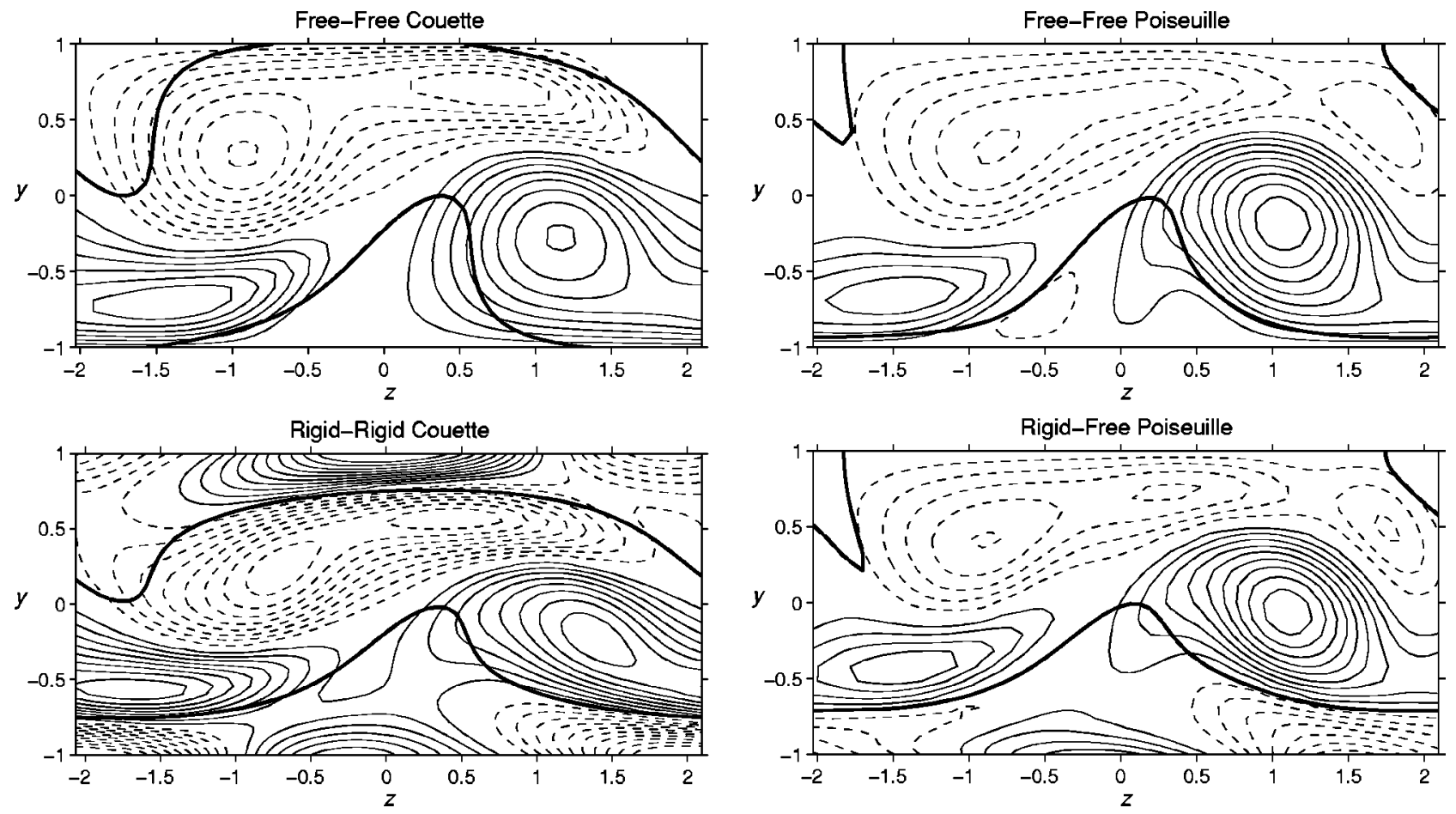

FIG. 10. Contours of streamwise vorticity $\omega_{x}$ at $\alpha x=3 \pi / 2$ for the same solutions as in Fig. 9. Equispaced levels at 0.1 max[ $\left.\omega_{x}(x, y, z)\right]$, except rigid-rigid Couette, where spacing is $0.1 \max \left[\omega_{x}(x, y=0, z)\right]$ (solid: positive, dash: negative). Thick lines are level curves $u=\min [u(x, y=0, z)]$ and $\max [u(x, y=0, z)]$. 

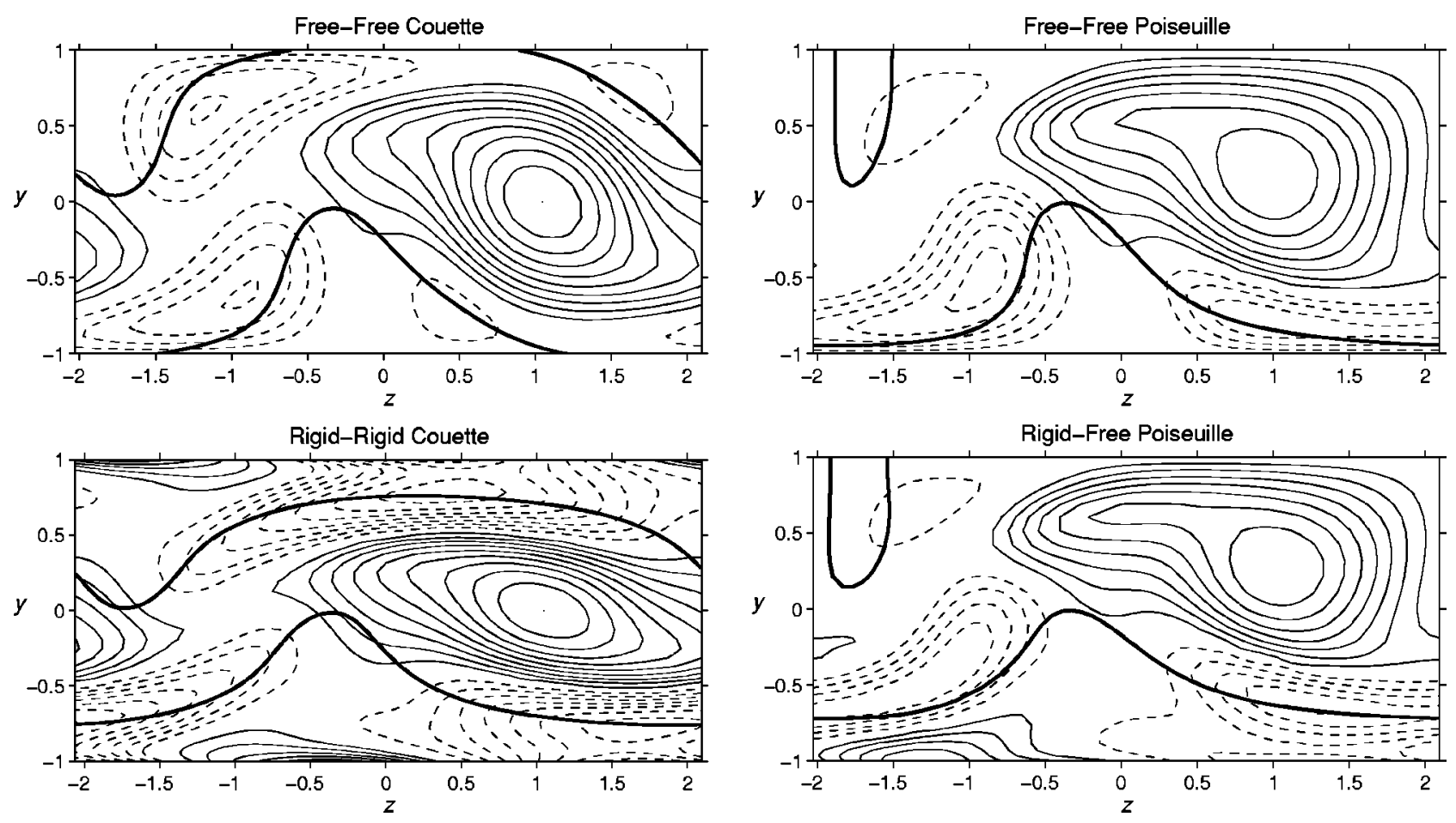

FIG. 11. Same as Fig. 10 but at $\alpha x=2 \pi$.

\section{OPTIMUM PARAMETERS}

The exact coherent structure solutions depend on three parameters: the horizontal wave numbers $\alpha$ and $\gamma$ and the Reynolds number Re, or, equivalently, the three length scales $L_{x}, L_{z}$, and $2 h$, nondimensionalized using wall units $\nu / u_{\tau}$,

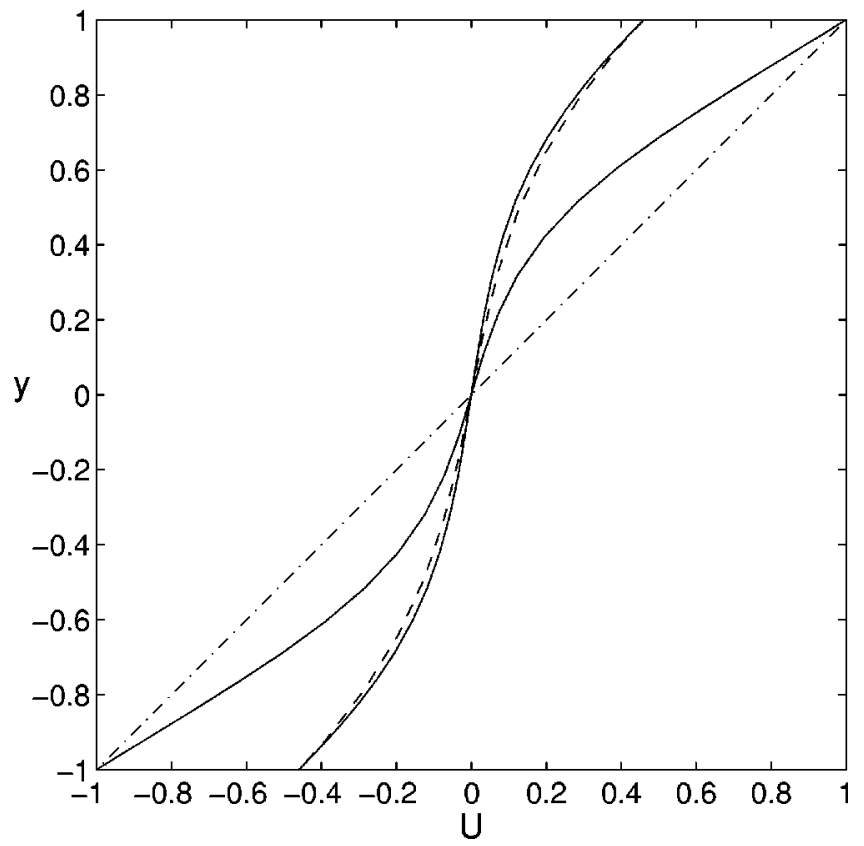

FIG. 12. Mean profiles for plane Couette flow. Laminar flow (dash-dot) and 3 -D steady states for $\alpha=0.5, \gamma=1.5$. No-slip solution at $\operatorname{Re}_{\mathrm{sn}}=163.4$, freeslip at $\operatorname{Re}_{\mathrm{sn}}=141.6$. Dash: no-slip solution with $y \rightarrow 1.5237 y$ to match freeslip wall velocities \pm 0.4598 . for instance, where $u_{\tau}$ is the friction velocity defined by $u_{\tau}^{2}$ $=\nu|d \bar{U} / d y|_{\text {wall }}$. The wave numbers $\alpha$ and $\gamma$ that lead to the lowest onset Reynolds number $\operatorname{Re}_{\mathrm{sn}}$ for the traveling wave solutions have been determined by minimizing the Reynolds number of the solutions over $\alpha, \gamma$, and $A_{x}$. The optimum wave numbers and corresponding minimum $\mathrm{Re}_{\mathrm{sn}}$ for "free-

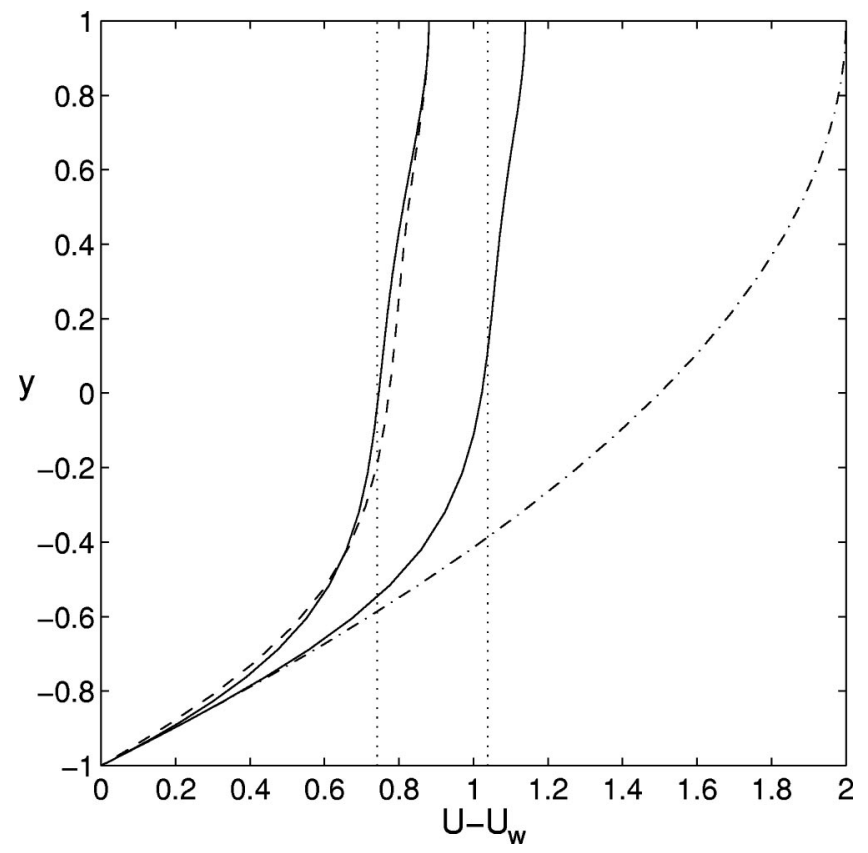

FIG. 13. Mean profiles for plane Poiseuille flow. Laminar flow (dash-dot) and 3-D traveling waves for $\alpha=0.5, \gamma=1.5$. Vertical dotted lines indicate wave velocity $C$. No-slip solution at $\operatorname{Re}_{\mathrm{sn}}=251.5, C=1.038$. Free-slip at $\operatorname{Re}_{\mathrm{sn}}=156.4, C=0.7413$. Dash: no-slip solution with $y \rightarrow 1+1.0721(y$ -1) to match free-slip wall velocities $U-U_{w}=0.8802$. 
free" plane Couette where $\operatorname{Re} \equiv\left(u_{\tau} \ell / \nu\right)^{2}$ have already been mentioned (Fig. 8). They are $\alpha=0.3236$ and $\gamma=0.7389$ yielding a minimum $\operatorname{Re}_{\mathrm{sn}}=99.98$. In wall units, these parameters correspond to $2 \ell^{+} \equiv 2 \ell u_{\tau} / \nu=2 \sqrt{\mathrm{Re}}=20, \quad L_{x}^{+}$ $\equiv \sqrt{\operatorname{Re}} L_{x} / \ell=194$, and $L_{z}^{+}=85$.

For rigid-rigid Couette where Re has the usual definition $\operatorname{Re} \equiv \mathcal{U} \ell / \nu$, where $\mathcal{U}$ is the half wall velocity difference and $\ell$ is the half-channel height, the optimum parameters are

$$
\alpha=0.577, \quad \gamma=1.150, \quad \operatorname{Re}_{\mathrm{sn}}=127.705 .
$$

That solution has wall shear rate $S \equiv|d \bar{U} / d y|_{\text {wall }}=1.809$, hence $u_{\tau} / \mathcal{U}=(1.809 / \mathrm{Re})^{1 / 2}$ so a length measured in wall units is $(1.809 \mathrm{Re})^{1 / 2}$ times the length in units of $\ell$ and

$$
2 \ell^{+} \equiv \frac{2 \ell u_{\tau}}{\nu}=30, \quad L_{x}^{+} \equiv 166, \quad L_{z}^{+}=83 .
$$

Clever and Busse ${ }^{27}$ performed a parametric study of threedimensional steady states in rigid-rigid Couette but they did not quote the optimum parameters (28).

For rigid-free Poiseuille, where the Reynolds number is defined such that $u_{\tau} \ell / \nu=(2 \mathrm{Re})^{1 / 2}$ with $\ell$ the half distance between the wall and the centerline, the optimum parameters are

$$
\alpha=0.5074, \quad \gamma=1.3165, \quad \operatorname{Re}_{\mathrm{sn}}=244.36,
$$

or in wall units which are simply $(2 \mathrm{Re})^{1 / 2}$ times the outer nondimensional units

$$
L_{x}^{+}=273.73, \quad L_{z}^{+}=105.51, \quad 2 \ell^{+}=44.21 .
$$

The optimization algorithm did not converge properly for the case of free-free Poiseuille flow. Approximate optimum parameters for free-free Poiseuille traveling waves where $u_{\tau} \ell / \nu=(2 \mathrm{Re})^{1 / 2}$ are $\alpha=0.38, \gamma=0.77, \mathrm{Re}_{\mathrm{sn}}=106$, or $L_{x}^{+}$ $=241, L_{z}^{+}=119,2 \ell^{+}=29$. The cause of these numerical problems may be that the length scales and amplitudes of the traveling wave solution increase as the Reynolds number decreases, hence the optimal solutions are more nonlinear and require finer resolutions in spite of the lower Reynolds numbers.

The most interesting results are those for rigid-free Poiseuille flow as those solutions may be relevant to the coherent structures observed near a (single) wall at higher Reynolds numbers. Indeed the optimum parameters (31) closely correspond to the observed scalings of the near-wall structures. The dependence of $\mathrm{Re}_{\mathrm{sn}}^{+}$on $L_{x}^{+}$and $L_{z}^{+}$for the rigidfree plane Poiseuille traveling waves is illustrated in Fig. 14. These results suggest that there are no traveling wave solutions below $L_{z}^{+} \approx 80$ or below $L_{x}^{+} \approx 200$. There exist traveling wave solutions for all larger $L_{x}$ and $L_{z}$ although the curves shown in Fig. 14 cannot be smoothly continued to arbitrarily large horizontal scales. The $\mathrm{Re}_{\mathrm{sn}}$ curve could not be smoothly continued beyond $L_{x}^{+} \approx 520$ (i.e., below $\alpha$ $=0.295$ ) for $\gamma=1.3$, for instance. The solution branches eventually develop a modulation as the length scales are increased and $\mathrm{Re}_{\mathrm{sn}}$ drops abruptly. This is undoubtedly linked to the fact that multiple copies of smaller scale traveling waves can fit in the domain if the latter is large enough (dashed lines in Fig. 14). The crossover between one- and
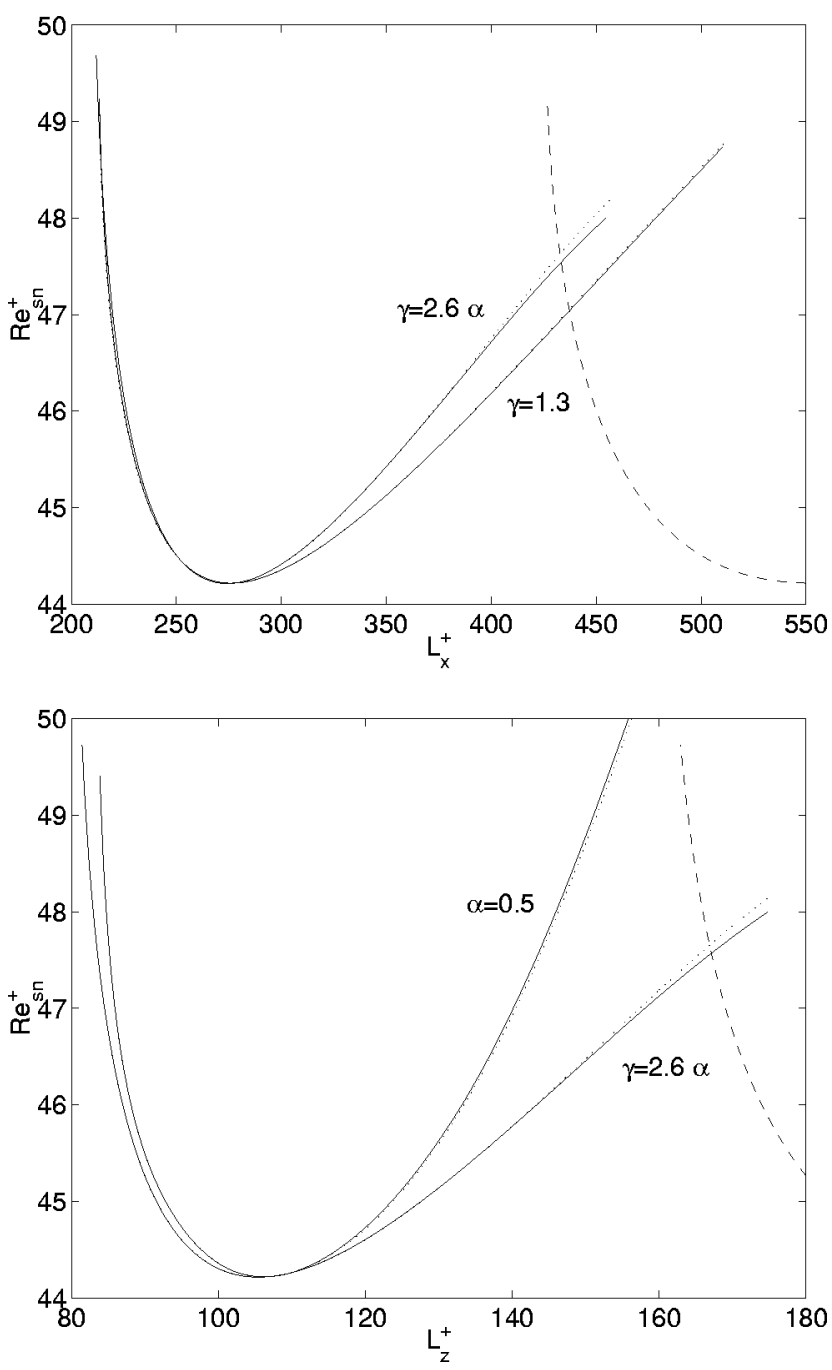

FIG. 14. Top: $\operatorname{Re}_{\mathrm{sn}}^{+}=2 \ell u_{\tau} / \nu$ as a function of $L_{x}^{+}$for $\gamma=1.3$ and $\gamma=2.6 \alpha$ for rigid-free plane Poiseuille flow traveling waves. Dash: 2-period solution for $\gamma=1.3$. Bottom: $\operatorname{Re}_{\mathrm{sn}}^{+}$as a function of $L_{z}^{+}$for $\alpha=0.5$ and $\gamma=2.6 \alpha$. Dash: 2-period solution for $\gamma=2.6 \alpha$. Solid: $\left(L_{T}, M_{T}, N_{T}\right)=(13,25,13)$; dot: $(11,23,11)$.

two-period solutions occurs at $L_{x}^{+} \approx 437$ for $\gamma=1.3$ and at $L_{z}^{+} \approx 167$ for domains with the aspect ratio $L_{x}=2.6 L_{z}$. Distinct traveling waves therefore arise at the same Reynolds number for those length scales. The optimum no-slip states are illustrated in Figs. 15 and 16.

\section{STATISTICS OF NO-SLIP ECS}

Figure 17 shows the normalized wall shear rate of the Poiseuille traveling wave at two wave number pairs $(\alpha, \gamma)$ as a function of the Reynolds number $\mathrm{Re}_{m}$ which is based on the bulk mean velocity and the full channel height. The 3-D traveling waves first arise at $\operatorname{Re}_{m} \approx 860$, which is significantly lower than the $\operatorname{Re}_{m}$ where turbulence is first observed. The Kim, Moin, and Moser ${ }^{16}$ turbulent flow was computed at $\mathrm{Re}_{m} \approx 5600$ while Toh and Itano, ${ }^{33,34}$ recently computed an asymmetric traveling wave at $\operatorname{Re}_{m}=4000$. The mean velocity profile and rms velocities normalized by $u_{\tau}$ are shown in Fig. 18 for the traveling wave with $\alpha=0.5$ and $\gamma=1.3$ on the upper branch at $\operatorname{Re}=473\left(\operatorname{Re}_{m}=1303\right)$, the turning point at 

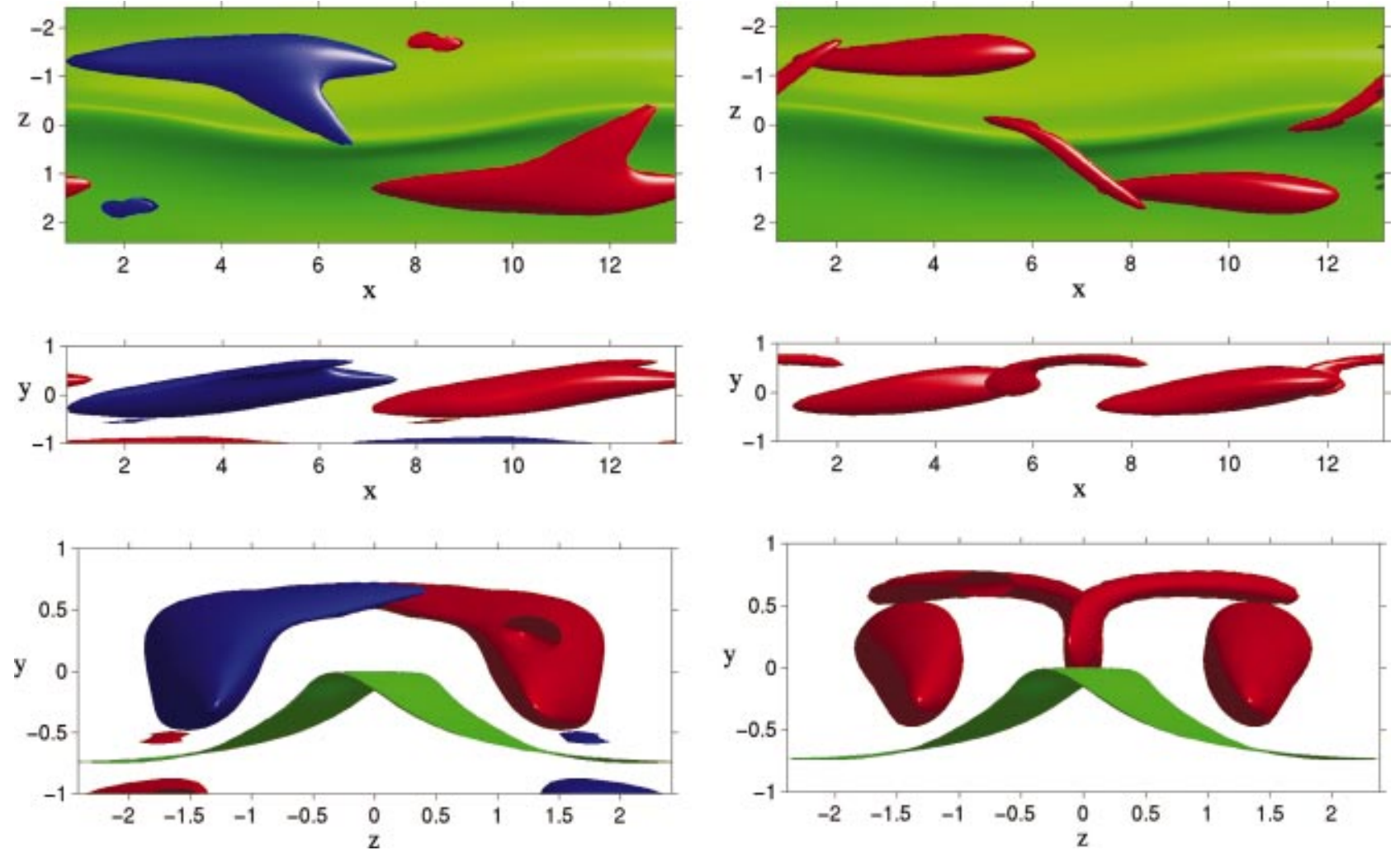

FIG. 15. (Color) Top, side, and back views of rigid-free plane Poiseuille flow traveling wave at its lowest friction Reynolds number $\left(2 \ell^{+}=44.21, L_{x}^{+}\right.$ $=273.73, L_{z}^{+}=105.51$ ). Green: Isosurface of streamwise velocity $u=\min [u(x, y=0, z)]$ (top and back views). Left column: Isosurfaces of streamwise vorticity at $\pm 0.6 \max \left(\omega_{x}\right)$ (red positive, blue negative). Right column: Red isosurfaces of $Q=0.40 Q_{\max }$, where $\nabla^{2} p=2 Q=W_{i j} W_{i j}-S_{i j} S_{i j}$ is twice the second invariant of the velocity gradient tensor. (Box shifted by $L_{x} / 16$.)

$\operatorname{Re}=244.41\left(\operatorname{Re}_{m}=867\right)$ and the lower branch at $\operatorname{Re}=454$ $\left(\mathrm{Re}_{m}=1390\right)$. The traveling wave rms velocities compare well with the rms velocities computed by Kim, Moin, and Moser (their Fig. 6) at the much higher $\operatorname{Re}_{m} \approx 5600\left(\operatorname{Re}_{\tau}\right.$ $\approx 180, \operatorname{Re}=\operatorname{Re}_{\tau}^{2} / 8 \approx 4050$ ). The main qualitative difference is that $v_{\text {rms }}$ is forced to go to zero at the channel centerline in the present traveling waves. This is probably directly connected, through a "splatting effect," with the increase in $w_{\text {rms }}$ near the centerline. Quantitatively, the levels of $u_{\text {rms }}$ are very close to their turbulent counterpart but the levels of $v_{\text {rms }}$ and $w_{\text {rms }}$ are lower. Hence, the traveling wave is more efficient at redistributing the streamwise velocity, i.e., at transporting momentum.

The normalized wall shear rate for the plane Couette steady states is shown in Fig. 19 as a function of the Reynolds number for several wave numbers $\alpha$ and $\gamma$. The upper branch of the $(\alpha, \gamma)=(0.5,1.5)$ solution shows a sharp turning point just before $\mathrm{Re}=400$. The solution branch turns back to lower Reynolds numbers then returns toward higher Reynolds numbers. Structural changes in the solution are subtle but one can detect the splitting of the streaks into two distinct, incomplete, streaks that are shifted by half a period in both horizontal directions (not shown in this paper). This suggests that solutions with wave numbers that are 1.5 or 2 times as large are favored at those Reynolds number for that horizontal aspect ratio. Indeed, solutions with $(\alpha, \gamma)$ $=(0.75,2.25)$ and $(1,3)$ exist and have higher wall shear rates at those Reynolds numbers (compare curves $\mathrm{c}, \mathrm{d}$, and $\mathrm{e}$ in Fig. 19). The complexity of the bifurcation diagram in the range $310 \leqslant \operatorname{Re} \leqslant 400$ is intriguing as this is precisely the range where turbulence is known to occur in plane Couette flow. ${ }^{21,35-37}$ For instance, in the (small) periodic box with $(\alpha, \gamma)=(0.5,1.5)$, there exist at least four distinct type of 3 -D steady states in $308<\operatorname{Re}<400$ and six distinct types in $329<\operatorname{Re}<400$. These lower bounds are very close to the $R_{u} \approx 312$ and $R_{c} \approx 323$ identified in much larger experimental domains. ${ }^{37}$

The mean velocity and rms velocity fluctuations of the plane Couette flow steady states are shown in Fig. 20. One significant difference with the Poiseuille flow traveling wave is that the lower branch mean velocity is closer to the laminar profile than the turning point profile. The lower branch shows very strong almost $x$-independent streaks at $\operatorname{Re}=400$ together with weak spanwise and wall-normal velocity fluctuations. This is in agreement with the scalings mentioned in Sec. IV A. The mean velocity and rms velocities of the upper branch solution at $\mathrm{Re}=400$ are very similar, qualitatively and quantitatively, with the turbulent Couette flow computed by Kawahara and Kida. ${ }^{38}$ The normalized wall shear rate $S$ in Fig. 19 is equal to the normalized energy input rate $I$ used by Kawahara and Kida. Their turbulent flow has $S=I \approx 3$ and this is precisely the level of $S$ seen for the upper branch steady solutions at $\mathrm{Re}=400$ (Fig. 19).

Kawahara and Kida managed to isolate an unstable timeperiodic solution in plane Couette flow that had been suggested by the work of Hamilton et al. ${ }^{14,21}$ Hamilton et al. 

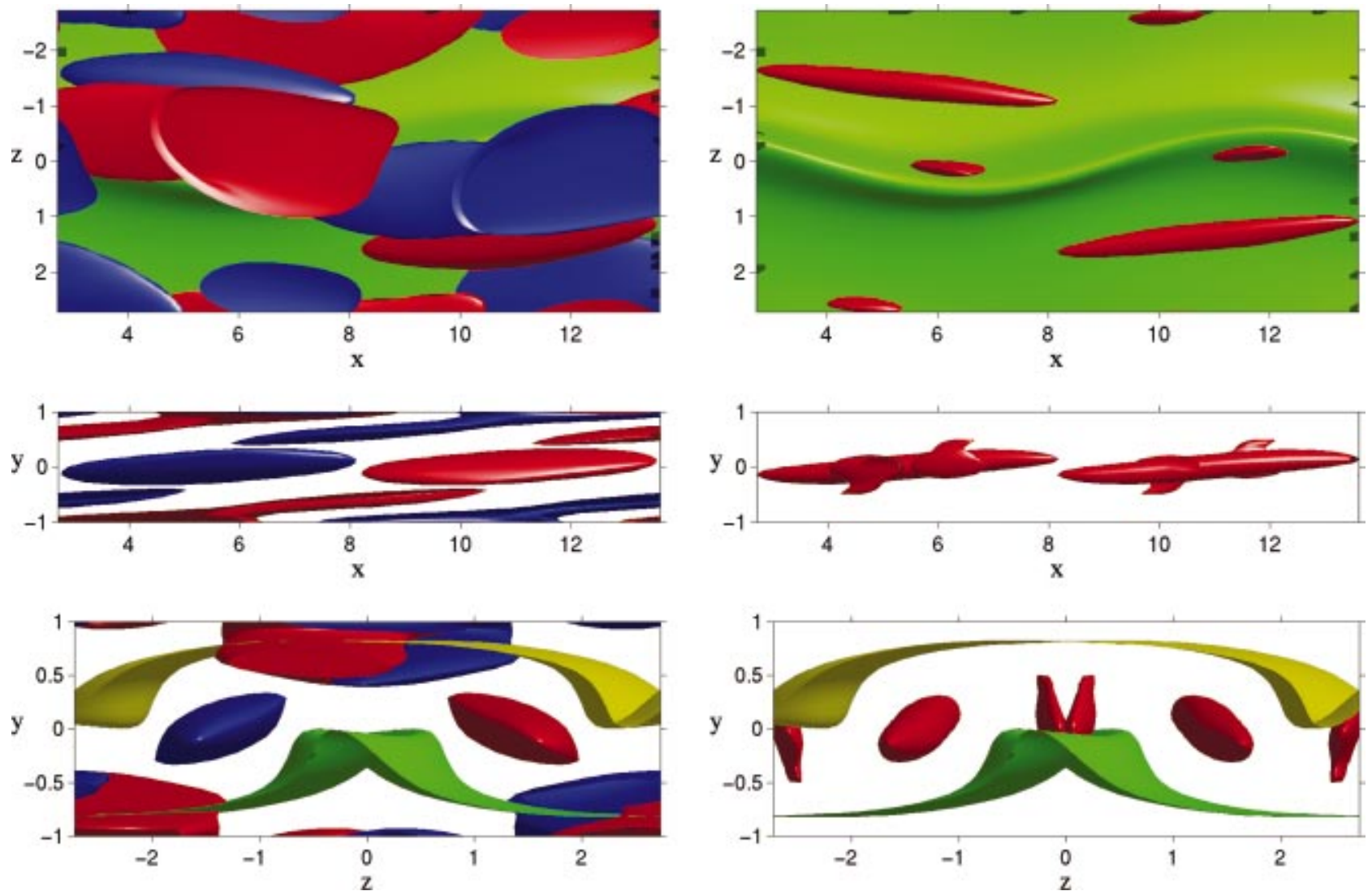

FIG. 16. (Color) Top, side, and back views of rigid-rigid plane Couette flow steady state at its lowest Reynolds number $(\alpha=0.5772, \gamma=1.1506$, Re $=127.7$ ). Green: Isosurface of streamwise velocity $u=\min [u(x, y=0, z)]$ (top and back views). Yellow: $u=\max [u(x, y=0, z)]$ (back view only). Left column: Isosurfaces of streamwise vorticity at $\pm 0.4 \max \omega_{x}$ (red positive, blue negative). Right column: Red isosurfaces are $Q=0.85 Q_{\max }$, where $\nabla^{2} p=2 Q$ $=W_{i j} W_{i j}-S_{i j} S_{i j}$ is twice the second invariant of the velocity gradient tensor. (Box shifted by $L_{x} / 4$.)

sought to isolate the self-sustaining process (Sec. IV A) by "quenching" of a turbulent flow. ${ }^{39,40}$ That trial-and-error procedure lead them to settle on the parameters $(\alpha, \gamma)$ $\approx(1.14,1.67)$, used by Kawahara and Kida. The present

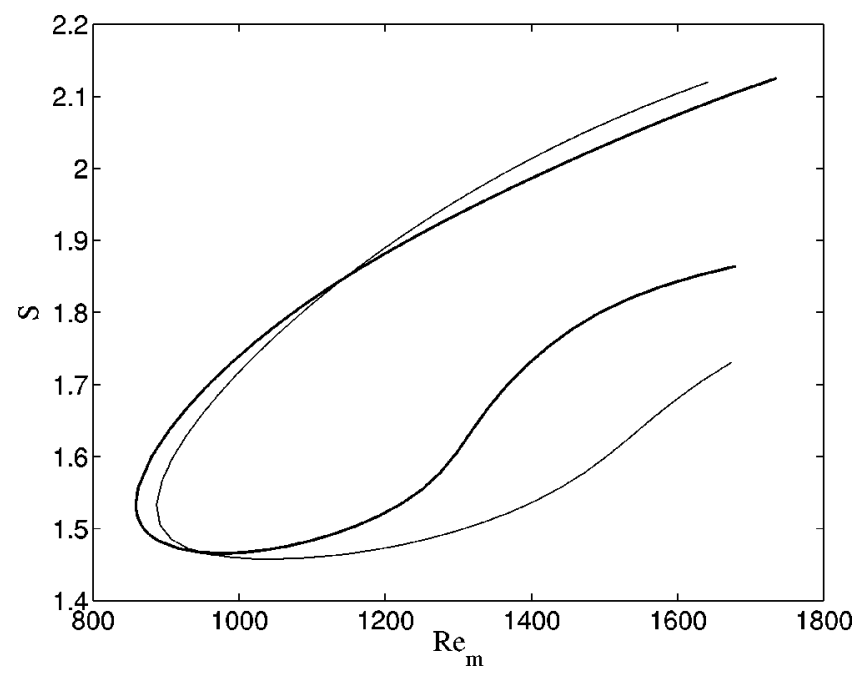

FIG. 17. Bifurcation diagrams for rigid-free Poiseuille. Wall shear rate, $S$, normalized by its laminar value vs bulk Reynolds number $\operatorname{Re}_{m}=\mathcal{U} H / \nu$ with $v=\left\langle U-U_{\text {wall }}\right\rangle$ and where $\mathcal{U}=\left\langle U-U_{\text {wall }}\right\rangle$ is the bulk mean velocity and $H$ $=4 \ell$ is the full channel height. $\operatorname{Re}_{m}=4 \mathcal{U} \operatorname{Re}$ and $S=4 /(3 \mathcal{U})$ in our units. Near optimum $(\alpha, \gamma)=(0.507,1.31)$ (thick curve) and $(\alpha, \gamma)=(0.5,1.5)$, $\left[L_{T}, M_{T}, N_{T}\right]=[13,25,13]$. study, and the earlier work of Clever and Busse, ${ }^{27}$ indicates that the 3-D steady states favor $\gamma / \alpha \approx 2$. This can be approximately understood in terms of the streak instability part of the self-sustaining process which consists essentially of a larger scale inflectional instability (e.g., Fig. 5 and Figs. 5 and 6 in Ref. 23). The streak instability also suggests, correctly, that at fixed $\gamma$ and Re, the spatial period-one solutions will disappear as $\alpha$ is increased. Further study is needed but this occurs apparently near $\gamma / \alpha \approx 1.5$. We have confirmed that there are indeed no spatial period-one solutions at $\mathrm{Re}$ $=400$ for $(\alpha, \gamma)=(1.14,1.67)(\gamma / \alpha=1.46)$, however there are solutions with $(\alpha, \gamma)=(0.84,1.67),(1.14,2.28)$ and $(1.14,3.34)$ for which the upper branch $S=I$ values at $\operatorname{Re}$ $=400$ are again in the neighborhood of 3 . Further detailed comparisons with the work of Kawahara and Kida will appear elsewhere.

\section{MODEL 1-D MAP}

The above-presented results demonstrate the remarkable qualitative and quantitative similarities between these 3-D traveling waves and structural as well as statistical features of turbulent shear flows. The major difference, of course, is that the traveling waves are steady in the proper Galilean frame and perfectly ordered while turbulent solutions are unsteady and disordered. An important characteristic of the traveling waves in this respect is that the traveling waves are 

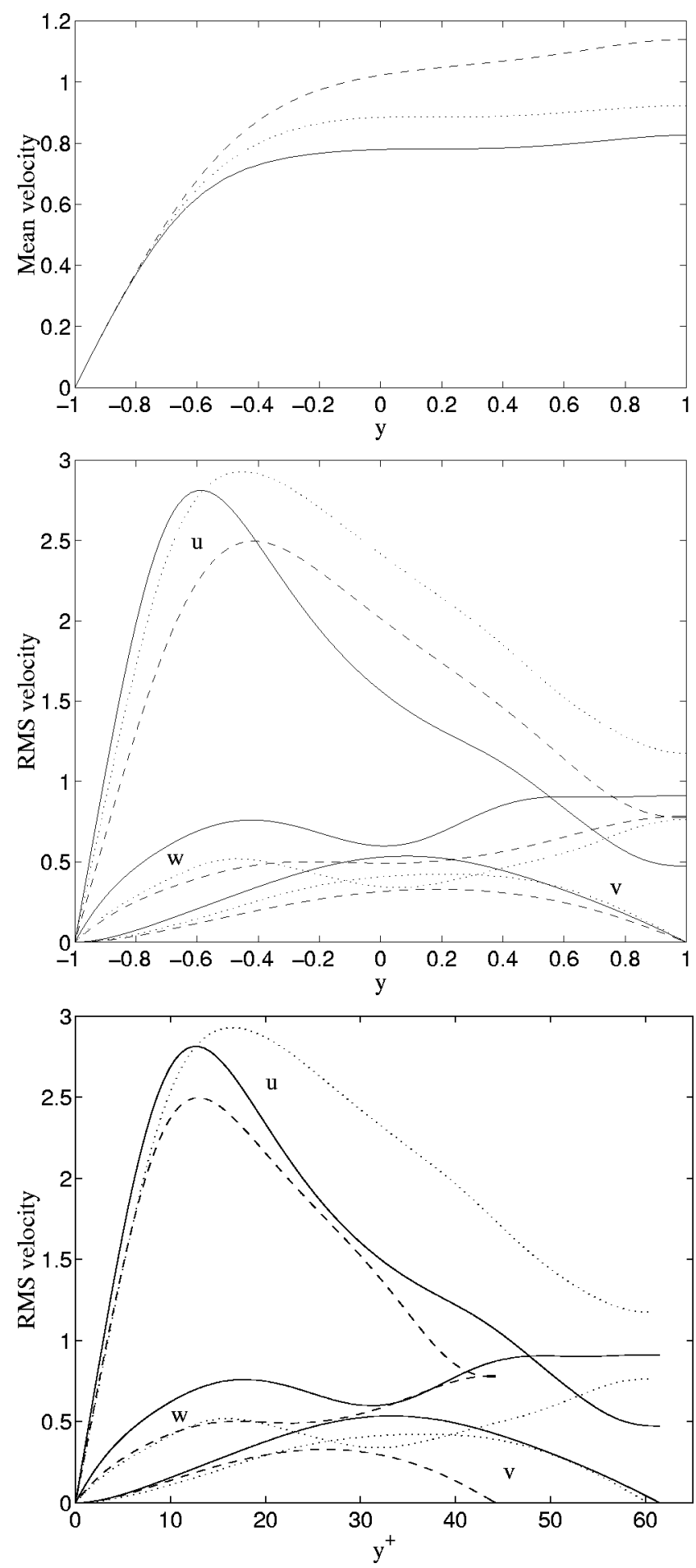

FIG. 18. Mean $\bar{U}(y)$ and rms velocities (normalized by $u_{\tau}$ ) for rigid-free Poiseuille traveling wave at $(\alpha, \gamma)=(0.5,1.3)$. Solid: Upper branch at $\operatorname{Re}$ $=473$. Dash: Turning point at $\operatorname{Re}_{\mathrm{sn}}=241.41$. Dot: Lower branch at $\operatorname{Re}$ $=454$. Bottom figure in wall units.

generally unstable from onset, except for a small range of stability of the plane Couette flow steady states reported in Ref. 27. A complete stability analysis of all the solutions presented here has not been performed, but selected stability results, and the lack of direct experimental or numerical observation of these solutions, suggests that these solutions are indeed typically unstable. This instability from onset is unusual and led to early skepticism about the relevance of such

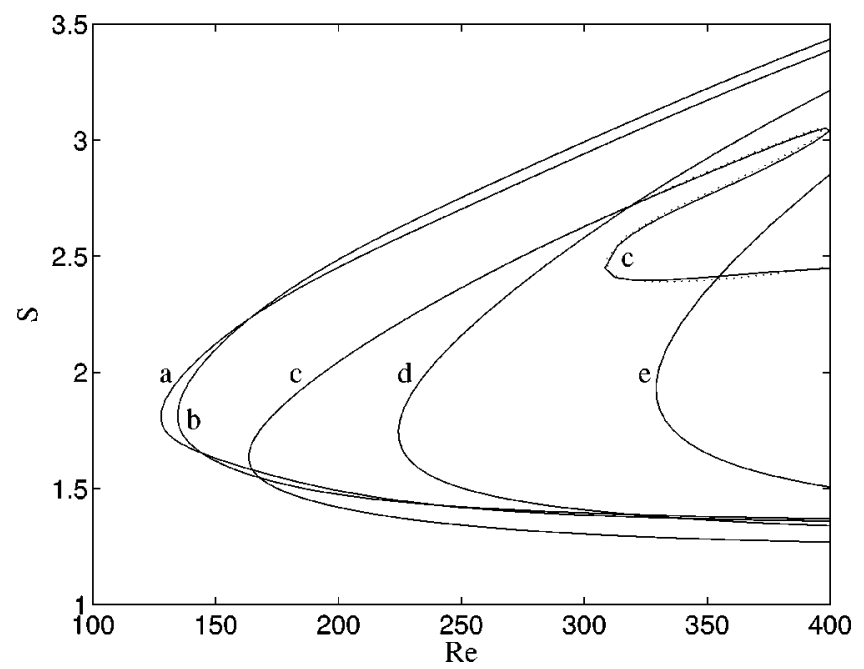

FIG. 19. Bifurcation diagrams for rigid-rigid Couette. Wall shear rate, $S$, normalized by its laminar value. (a) $(\alpha, \gamma)=(0.5772,1.1506)$, $\left[L_{T}, M_{T}, N_{T}\right]=[15,27,15]$ and $[13,25,13]$ overlap. (b) $(\alpha, \gamma)=(0.75,1.5)$, $[13,25,13]$ and $[11,13,11]$ overlap. (c) $(\alpha, \gamma)=(0.5,1.5),[15,27,15]$ and $[13,25,13]$ (dot). (d) $(\alpha, \gamma)=(0.75,2.25),[13,27,13]$. (e) $(\alpha, \gamma)=(1,3)$, $[13,27,13]$

solutions, ${ }^{41}$ when the connections with observed coherent structures had not been revealed. However, others (e.g., Refs. $42,34,20,38)$ see this instability of the exact coherent structures as a feature, not a bug. Indeed, turbulence is often described as a "cascade of instabilities." Whatever the exact nature, and validity, of that cascade of instabilities concept, it is related certainly not to the stable laminar state but much more likely to the instabilities of the exact coherent states.

A simple 1-D map $x_{n+1}=f_{\mu}\left(x_{n}\right)$ illustrates these features of the onset of shear turbulence and the possible connections between turbulence and the unstable exact coherent states. The map is defined by (Fig. 21)

$$
f_{\mu}(x)=\left\{\begin{array}{ccc}
\mu \frac{x}{1-x} & \text { if } & 0 \leqslant x \leqslant \frac{1}{2} \\
f_{\mu}(1-x) & \text { if } \frac{1}{2}<x \leqslant 1 .
\end{array}\right.
$$

The parameter $0 \leqslant \mu \leqslant 1$ is directly related to the Reynolds number, e.g. $\mu=\operatorname{Re} /\left(\operatorname{Re}+\operatorname{Re}_{c}\right)$ for some critical Reynolds number $\operatorname{Re}_{c}$, with $\mu=0$ corresponding to $\operatorname{Re}=0$ and $\mu=1$ to $\operatorname{Re}=\infty$.

The map is such that $x=0$ is the laminar fixed point, stable for all $0 \leqslant \mu<1$ and neutrally stable in the limit $\mu$ $=1(\operatorname{Re} \rightarrow \infty)$, because $f^{\prime}(0)=\mu$.

The map (32) bears some algebraic resemblance to the famous logistic map ${ }^{43} f(x)=\mu x(1-x)$ that shows the period doubling route to chaos (see, e.g., Ref. 44), but the present map has a cusp at $x=1 / 2$, where $f(1 / 2)=\mu$ is the maximum value of $f(x)$. This cusp is necessary to yield a bifurcation to new nontrivial fixed points that are unstable from onset, as is the case for the map (32) where new unstable fixed points exist for $\mu>1 / 2$. The new fixed points are an upper branch $x_{u}$ and a lower branch $x_{\ell}$, 

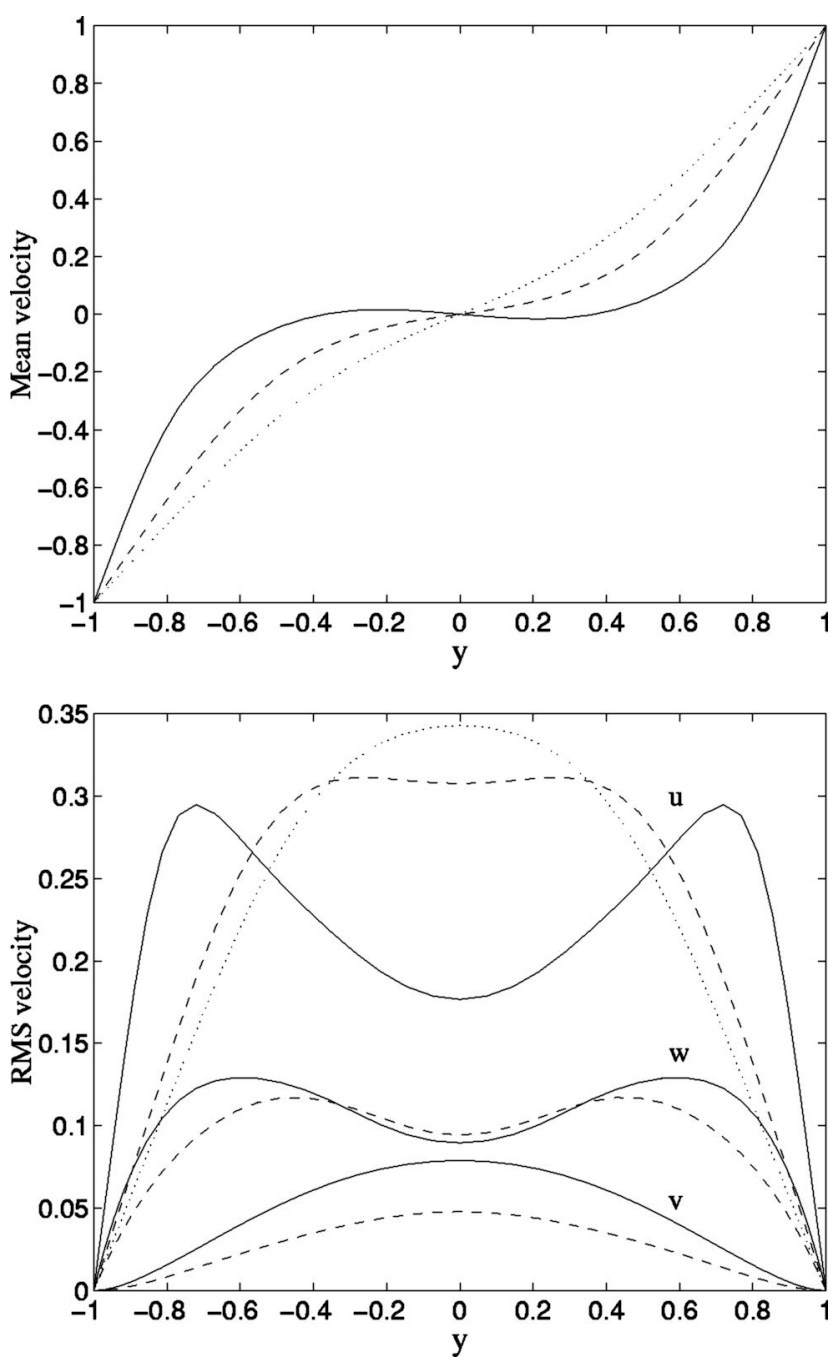

FIG. 20. Mean $\bar{U}(y)$ and rms velocities for rigid-rigid plane Couette steady states with $(\alpha, \gamma)=(0.5772,1.1506)$. Solid: Upper branch at $\operatorname{Re}=400$. Dash: Turning point at $\operatorname{Re}_{\mathrm{sn}}=127.7$. Dot: Lower branch at $\operatorname{Re}=400[v, w$ are much smaller and omitted, $\max (v)=0.0075, \max (w)=0.02]$.

$$
x_{u}=\frac{\sqrt{\mu^{2}+4 \mu}-\mu}{2}, \quad x_{\ell}=1-\mu .
$$

Unstable periodic orbits of all periods also exist when $\mu$ $>1 / 2$. The period-2 solution oscillates between $x_{1}$ and $x_{2}$ $=f_{\mu}\left(x_{1}\right)$ with $x_{1}<1 / 2<x_{2}$ and

$$
x_{1}=\frac{\sqrt{(\mu+1)^{2}+4}-(\mu+1)}{2} .
$$

More significantly, two period-3 solutions exist for $\mu>1 / 2$. This implies the existence of periodic orbits of all periods by Sarkovskii's theorem. ${ }^{45}$ The period-3 solutions consist of an "outer" solution (dash in Fig. 21) that oscillates between $x_{1}$, $x_{2}=f_{\mu}\left(x_{1}\right), x_{3}=f_{\mu}\left(x_{2}\right)$ with $x_{1}<x_{2}<1 / 2<x_{3}$ and

$$
x_{1}=\frac{\sqrt{A^{2}+4 \mu}-A}{2 \mu}, \quad A=\mu^{2}+\mu+1
$$

and an "inner" period-3 solution (dash-dot in Fig. 21) oscillating between $x_{1}, x_{2}=f_{\mu}\left(x_{1}\right), x_{3}=f_{\mu}\left(x_{2}\right)$ with $x_{1}<1 / 2$ $<x_{2}<x_{3}$ and

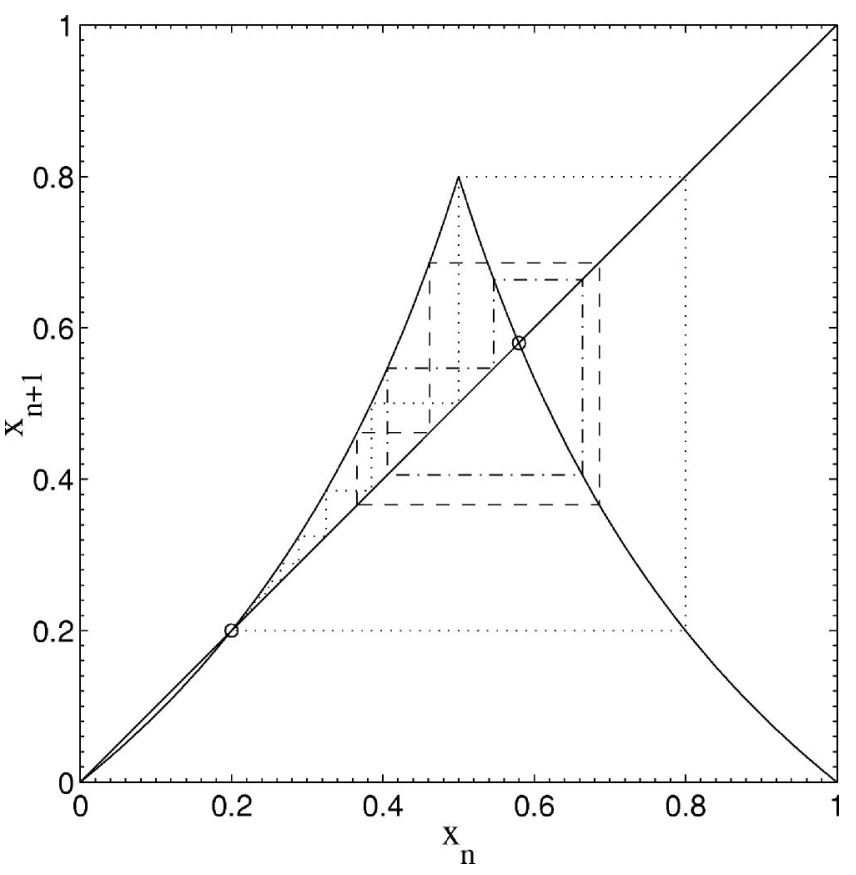

FIG. 21. The map (32) for $\mu=0.8$ with the unstable fixed points $x_{\ell}$ and $x_{u}$ (open circles), period-3 solutions (dash and dash-dot) and the homoclinic orbit $x_{n-1}=\left(1+\mu / x_{n}\right)^{-1}, x_{0}=1 / 2, \mu, 1-\mu=x_{\ell}$ (dot).

$$
x_{1}=\frac{\sqrt{B^{2}+4 \mu(\mu+1)}-B}{2(\mu+1)}, \quad B=\mu^{2}+2 \mu-1 .
$$

Finally, there is a homoclinic orbit connecting the lower branch fixed point $x_{\ell}$ to itself when $\mu>1 / 2$. That orbit, depicted by a dotted line in Fig. 21, consists of $x_{0}=1 / 2$ with $x_{1}=f_{\mu}\left(x_{0}\right)=\mu$ and $x_{2}=f_{\mu}\left(x_{1}\right)=1-\mu=x_{\ell}$ and the preiterates of $x_{0}$,

$$
x_{n-1}=\frac{1}{1+\mu / x_{n}}, \quad n \leqslant 0 .
$$

There are infinitely many other distinct homoclinic orbits.

It is clear (Fig. 21) that for all initial conditions in the interval $[1-\mu, \mu], x_{n}$ stays within that interval for all $n$, yet there are no stable fixed points or periodic orbits there. Hence, when $\mu$ crosses $1 / 2$, i.e., Re becomes larger than the critical value $\mathrm{Re}_{c}$, there is a sudden transition to a truly chaotic regime for almost all initial conditions in that interval. The dynamics for almost all initial conditions in the open interval $] 1-\mu, \mu[$ when $\mu>1 / 2$ consists of an aperiodic oscillation about the upper branch $x_{u}$ with excursions toward the largest value $x=\mu$ followed by collapse toward the lower branch $x_{\ell}$ then a climb back toward $x_{u}$, and so on. Therefore, one may expect that the upper branch $x_{u}$ provides a good first approximation for the average $x_{n}$.

The 1-D map (32) thus illustrates how the onset of unstable periodic solution may be directly related to a transition to "turbulence" and why the upper branch solutions may offer a good first approximation to the mean properties of the turbulence. That simple map has not been directly connected to the Navier-Stokes dynamics but one should note the resemblance with the "Lorenz map" which was deduced from a continuous differential system. 
Note that in the discrete map, the fixed points $x_{\ell}$ and $x_{u}$ may correspond to fixed points or traveling waves as in this paper or to fundamental periodic solutions of the Navier-Stokes equations as in Ref. 38.

The simple map (32) has the particularity that the maximum value $f(1 / 2)=\mu$ is the pre-iterate of the lower branch $x_{\ell}$ as shown in Fig. 21. This is the feature leading to the existence of homoclinic orbits. This map therefore is just on the border between two types of maps. This map and slightly broader maps lead to strange attractors, but slightly spikier maps for which the pre-iterate of $x_{\ell}$ is less than $\mu$ will lead to a strange repellor (see the discussions of the logistic map with $\mu>4$ in Ref. 45 or 44, exercise 11.4.6). For the spikier maps, almost all initial conditions will eventually end up converging to the laminar point $x=0$. A concrete example is the two-parameter family of maps defined by

$$
f(x)= \begin{cases}\frac{\mu x}{1-2^{\epsilon} x^{1+\epsilon}} & \text { if } 0 \leqslant x \leqslant \frac{1}{2}, \\ f(1-x) & \text { if } \frac{1}{2}<x \leqslant 1,\end{cases}
$$

where $-1<\epsilon$ and $0 \leqslant \mu<1$. These maps have the same overall shape and properties as the map (32), in particular, $f(1 / 2)=\mu$ and $f^{\prime}(0)=\mu$ if $-1<\epsilon$. The additional parameter $\epsilon$ controls the spikiness. The maps are spikier than (32) if $0<\epsilon$. When $\epsilon=-1,(38)$ becomes the tent map. ${ }^{44,45}$ If $-1<\epsilon \leqslant 0$ and $\mu>1 / 2$, all initial conditions in $\left[x_{\ell}, 1-x_{\ell}\right]$ are trapped in that interval yet there are no stable periodic points there. If $\epsilon>0$ and $\mu>1 / 2$, a strange repellor exists. Almost all initial conditions, except for a Cantor set, ${ }^{45}$ will eventually end up at the laminar fixed point $x=0$. An imperceptible change in the shape of the map, from a small negative $\epsilon$ to a small positive $\epsilon$, can lead to drastic differences for the long time behavior of the dynamical system. This provides another concrete illustration that may be useful also for shear flows where there is some limited evidence for strange repellors, from low-order truncations of the Navier-Stokes equations. ${ }^{42}$ It may be that small periodic domains lead to strange repellors but slightly larger domains lead to strange attractors. Finally, one can imagine a more complex scenario, e.g., $\epsilon=3 / 4-\mu$, where the map broadens with increasing $\mu$, and the transition to a strange attractor occurs at $\mu=3 / 4$, well beyond the onset of unstable fixed points and periodic orbits at $\mu=1 / 2$.

\section{CONCLUDING REMARKS}

Three-dimensional traveling wave solutions of the Navier-Stokes equations have been calculated for plane Couette and Poiseuille flows with both free-slip and no-slip boundary conditions. These well-resolved solutions capture essential structural and statistical features of turbulent shear flows and are therefore called exact coherent structures. These exact coherent structures are typically unstable from their onset at a Reynolds number that is about twice lower than those where turbulence is first observed. It is believed that the inherent instabilities of the exact coherent structure are directly related to the disorder characteristic of turbulent shear flows. The exact coherent structures and their associ- ated instabilities are thus good candidates for precise definitions of Townsend's notions of active and inactive motions.

The initial step to calculate these exact coherent structures is to track three-dimensional solutions that bifurcate from a 2-D streaky flow. This step demonstrates the validity of a previously studied self-sustaining process and establishes a direct link between self-sustained 3-D coherent structures and Townsend's attached eddies. The next steps consist of homotopy transformations that demonstrate the close relationships between the various solutions.

The traveling waves come in pairs above their onset Reynolds number. Our simplistic view is that the lower branch and its stable manifold form the separatrix between the basin of attraction of the laminar state and the turbulent domain in phase space. This is similar to the view of Toh and Itano, ${ }^{33,34}$ who propose that motion along the unstable manifold of the lower branch corresponds to bursting. Toh and Itano do not distinguish between upper branch and lower branch solutions however. This may be because their shooting approach, similar to our method of bifurcation from 2-D streaky flow, may be limited to capturing the lower branch solution as suggested in Ref. 20. We propose further that the turbulent state is essentially an aperiodic oscillation about the upper branch solution. Therefore the upper branch provides a good first approximation to the turbulence statistics. It is unclear then whether "bursting" would correspond to excursions along the unstable manifold of the lower or the upper branch. This picture of the nature of shear turbulence has been illustrated by a simple 1-D map. Jimenez and Simens ${ }^{47}$ also compute a single traveling wave solution in a plane Poiseuille flow where vorticity fluctuations are wiped out beyond a pre-set distance from the wall. It is unclear whether that solution corresponds to an upper or lower branch.

Schmiegel, Eckhardt, and Mersmann ${ }^{42,48}$ have suggested that the number of unstable steady states in Couette-like flow grows with the Reynolds number and that shear turbulence may consist of a chaotic repellor that forms around heteroclinic connections between those steady states. Our own related studies of "low"-order models (Sahay and Waleffe, 2000 , unpublished) extending our earlier work ${ }^{23}$ show similar results, however we believe that most of those steady states are spurious solutions that do not converge to solutions of the Navier-Stokes equations. The number of those steady states seems to grow with both the Reynolds number and the resolution, therefore this is an intriguing numerical issue. Nonetheless, the idea of the onset of a multitude of unstable states is likely to be relevant, with most unstable states corresponding not to steady states but to periodic orbits, and the strange repellor idea may also be applicable in some range of parameters. There is solid evidence for unstable periodic solutions in plane Couette flow ${ }^{38}$ in addition to the steady states presented here and in earlier references. ${ }^{25,27}$ There is an obvious structural and statistical connection between those solutions but the phase space connections have not been elucidated yet. It appears that periodic solutions may replace the steady states in domains where the streamwise period is less than about 1.6 times the spanwise period (at low Reynolds numbers of course). Clever and Busse ${ }^{27}$ had already reported 
existence of a time periodic solution in plane Couette flow, but this appears to be a different solution than that found by Kawahara and Kida. Further work needs to be done to elucidate the connections between these various solutions and turbulent flow.

In any case, one may hope that the knowledge of all these solutions will lead to a full understanding of the onset and nature of turbulence, at least in plane Couette flow. There are intriguing connections between critical Reynolds number values found here for steady states in plane Couette flow and physical experiments in much larger domains. ${ }^{37} \mathrm{In}$ fact, we believe that most of the characteristics of turbulence can be captured in relatively small periodic domains and that other features such as spots and "barber pole" structure ${ }^{49}$ are "secondary" spatio-temporal complexities. Finally, it is most likely that the steady states and traveling wave solutions discussed here can be extended to traveling waves in pipe flow and to self-similar solutions in mixing layers.

The data presented in this paper are available from the author by request. The data will eventually be posted at http://www.math.wisc.edu/ waleffe/ECS/.

\section{ACKNOWLEDGMENTS}

The author is grateful for support through NSF-DMS9803685 and NSF-DMS-0204636. Most of the computations were performed on a SGI origin 200 purchased with SCREMS Award No. NSF-DMS-9977384.

${ }^{1}$ T. Theodorsen, "Mechanism of turbulence," in Proceedings of the Second Midwestern Conference on Fluid Mechanics, Ohio State University, 1982, pp. $1-18$.

${ }^{2}$ T. Theodorsen, "The structure of turbulence," in Fluid Dynamics and Applied Mathematics, edited by J.B. Diaz and S.I. Pai (Gordon and Breach, New York, 1962), pp. 21-27.

${ }^{3}$ W.V.R. Malkus, "Outline of a theory of turbulent shear flow," J. Fluid Mech. 1, 521 (1956).

${ }^{4}$ F.H. Busse, "Bounds for turbulent shear flow," J. Fluid Mech. 41, 219 (1970).

${ }^{5}$ A.A. Townsend, Structure of Turbulent Shear Flow (Cambridge University Press, Cambridge, 1956), pp. 315.

${ }^{6}$ A.A. Townsend, The Structure of Turbulent Shear Flow, 2nd ed. (Cambridge University Press, Cambridge, 1976).

${ }^{7}$ S.J. Kline, W.C. Reynolds, F.A. Schraub, and P.W. Rundstadler, "The structure of turbulent boundary layers," J. Fluid Mech. 30, 741 (1967).

${ }^{8}$ C.R. Smith and S.P. Metzler, "The characteristics of low-speed streaks in the near-wall region of a turbulent boundary layer," J. Fluid Mech. 129, 27 (1983).

${ }^{9}$ Self-Sustaining Mechanisms of Wall Turbulence, edited by R.L. Panton (Computational Mechanics, Southampton, 1997).

${ }^{10} \mathrm{P}$. Holmes, J.L. Lumley, and G. Berkooz, Turbulence, Coherent Structures, Dynamical Systems, and Symmetry (Cambridge University Press, Cambridge, 1996).

${ }^{11}$ M.S. Acarlar and C.R. Smith, "A study of hairpin vortices in a laminar boundary layer," J. Fluid Mech. 175, 1 (1987); 175, 43 (1987).

${ }^{12}$ D.J. Benney, "The evolution of disturbances in shear flows at high Reynolds numbers," Stud. Appl. Math. 70, 1 (1984).

${ }^{13} \mathrm{~F}$. Waleffe, "Organized motions underlying turbulent shear flows," Annual Research Briefs-1989, Center for Turbulence Research, Stanford University, 1989, pp. 107-115.

${ }^{14} \mathrm{~F}$. Waleffe, J. Kim, and J. Hamilton, "On the origin of streaks in turbulent shear flows," in Turbulent Shear Flows 8: Selected Papers from the Eighth International Symposium on Turbulent Shear Flows, Munich, Germany, 9-11 September 1991, edited by F. Durst, R. Friedrich, B.E. Launder, F.W. Schmidt, U. Schumann, and J.H. Whitelaw (Springer, Berlin, 1993), pp. 37-49.

${ }^{15}$ D.D. Stretch, "Automated pattern eduction from turbulent flow diagnostics," Annual Research Briefs-1990, Center for Turbulence Research, Stanford University, pp. 145-157.

${ }^{16}$ J. Kim, P. Moin, and R.D. Moser, "Turbulence statistics in fully developed channel flow at low Reynolds number," J. Fluid Mech. 177, 133 (1987).

${ }^{17}$ J. Jeong, F. Hussain, W. Schoppa, and J. Kim, "Coherent structures near the wall in a turbulent channel flow," J. Fluid Mech. 332, 185 (1997).

${ }^{18}$ R.J. Adrian, C.D. Meinhart, and C.D. Tomkins, "Vortex organization in the outer region of the turbulent boundary layer," J. Fluid Mech. 422, 1 (2000).

${ }^{19} \mathrm{~F}$. Waleffe, "Three-dimensional coherent states in plane shear flows," Phys. Rev. Lett. 81, 4140 (1998).

${ }^{20} \mathrm{~F}$. Waleffe, "Exact coherent structures in channel flow," J. Fluid Mech. 435, 93 (2001).

${ }^{21}$ J.M. Hamilton, J. Kim, and F. Waleffe, "Regeneration mechanisms of near-wall turbulence structures," J. Fluid Mech. 287, 317 (1995).

${ }^{22} \mathrm{~F}$. Waleffe, "Hydrodynamic stability and turbulence: Beyond transients to a self-sustaining process," Stud. Appl. Math. 95, 319 (1995).

${ }^{23} \mathrm{~F}$. Waleffe, "On a self-sustaining process in shear flows," Phys. Fluids 9, 883 (1997)

${ }^{24}$ D.D. Joseph and L.N. Tao, "Transverse velocity components in fully developed unsteady flows," J. Appl. Phys. 30, 147 (1963).

${ }^{25}$ M. Nagata, "Three-dimensional finite-amplitude solutions in plane Couette flow: Bifurcation from infinity," J. Fluid Mech. 217, 519 (1990).

${ }^{26}$ R.M. Clever and F.H. Busse, "Three-dimensional convection in a horizontal layer subjected to constant shear," J. Fluid Mech. 234, 511 (1992).

${ }^{27}$ R.M. Clever and F.H. Busse, "Tertiary and quaternary solutions for plane Couette flow," J. Fluid Mech. 344, 137 (1997).

${ }^{28}$ J.D. Murray, Mathematical Biology (Springer, New York, 1993), pp. 767.

${ }^{29}$ L. Greengard, "Spectral integration of two-point boundary value problem," SIAM (Soc. Ind. Appl. Math.) J. Numer. Anal. 28, 1071 (1991).

${ }^{30} \mathrm{~F}$. Waleffe and J. Kim, "How streamwise rolls and streaks self-sustain in a shear flow," in Self-Sustaining Mechanisms of Wall Turbulence, edited by R.L. Panton (Computational Mechanics, Southampton, 1997), pp. 385422.

${ }^{31} \mathrm{~F}$. Waleffe and J. Kim, "How streamwise rolls and streaks self-sustain in a shear flow: Part 2," AIAA Pap. 98-2997 (1998).

${ }^{32}$ S.C. Reddy, P.J. Schmid, J.S. Baggett, and D.S. Henningson, “On stability of streamwise streaks and transition thresholds in plane channel flows," J. Fluid Mech. 365, 269 (1998).

${ }^{33} \mathrm{~S}$. Toh and T. Itano, "Low-dimensional dynamics embedded in a plane Poiseuille flow turbulence: Traveling-wave solution is a saddle point?" xxx.lanl.gov/abs/physics/9905012 (1999).

${ }^{34} \mathrm{~T}$. Itano and S. Toh, "The dynamics of bursting process in wall turbulence," J. Phys. Soc. Jpn. 70, 701 (2001).

${ }^{35}$ N. Tillmark and P.H. Alfredsson, "Experiments on transition in plane Couette flow," J. Fluid Mech. 235, 89 (1992).

${ }^{36}$ O. Dauchot and F. Daviaud, "Finite amplitude perturbation and spots growth mechanism in plane Couette flow," Phys. Fluids 7, 335 (1995).

${ }^{37}$ S. Bottin, F. Daviaud, P. Manneville, and O. Dauchot, "Discontinuous transition to spatiotemporal intermittency in plane Couette flow," Europhys. Lett. 43, 171 (1998)

${ }^{38}$ G. Kawahara and S. Kida, "Periodic motion embedded in plane Couette turbulence: Regeneration cycle and burst," J. Fluid Mech. 449, 291 (2001).

${ }^{39}$ J. Jimenez and P. Moin, "The minimal flow unit in near-wall turbulence," J. Fluid Mech. 225, 213 (1991).

${ }^{40}$ A. Schmiegel and B. Eckhardt, "Persistent turbulence in annealed plane Couette flow," Europhys. Lett. 51, 395 (2000).

${ }^{41}$ A. Lundbladh and A. Johansson, "Direct simulation of turbulent spots in plane Couette flow," J. Fluid Mech. 229, 499 (1991).

${ }^{42}$ A. Schmiegel and B. Eckhardt, "Fractal stability border in plane Couette flow," Phys. Rev. Lett. 79, 5250 (1997).

${ }^{43}$ R.M. May, "Simple mathematical models with very complicated dynamics," Nature (London) 261, 459 (1976).

${ }^{44}$ S.H. Strogatz, Nonlinear Dynamics and Chaos (Addison-Wesley, Reading, MA, 1994).

${ }^{45}$ R.L. Devaney, An Introduction to Chaotic Dynamical Systems, 2nd ed. (Addison-Wesley, Redwood City, CA, 1989).

${ }^{46}$ E.N. Lorenz, "Deterministic nonperiodic flow," J. Atmos. Sci. 20, 130 (1963).

${ }^{47}$ J. Jimenez and M. Simens, "Low-dimensional dynamics of a turbulent wall flow," J. Fluid Mech. 435, 81 (2001).

${ }^{48}$ B. Eckhardt and A. Mersmann, "Transition to turbulence in a shear flow," Phys. Rev. E 60, 509 (1999).

${ }^{49}$ F. Prigent, G. Gregoire, H. Chate, O. Dauchot, and W. Van Saarloos, "Large-scale finite-wavelength modulation within turbulent shear flows," Phys. Rev. Lett. 89, 014501 (2002). 